\title{
RAF1 amplification drives a subset of bladder tumors and confers sensitivity to MAPK-directed therapeutics
}

\author{
Raie T. Bekele, ${ }^{1,2}$ Amruta S. Samant, ${ }^{1}$ Amin H. Nassar, ${ }^{3,4}$ Jonathan So, ${ }^{4}$ Elizabeth P. Garcia, ${ }^{3}$ Catherine R. Curran, ${ }^{4}$ Justin H. Hwang, ${ }^{2,4}$ \\ David L. Mayhew, ${ }^{2,4}$ Anwesha Nag, ${ }^{5}$ Aaron R. Thorner, ${ }^{5}$ Judit Börcsök, ${ }^{6}$ Zsofia Sztupinszki, ${ }^{6}$ Chong-Xian Pan, ${ }^{7}$ Joaquim Bellmunt, ${ }^{8}$ \\ David J. Kwiatkowski, ${ }^{2,3}$ Guru P. Sonpavde, ${ }^{4}$ Eliezer M. Van Allen, ${ }^{2,4}$ and Kent W. Mouw ${ }^{1,2,3}$

\begin{abstract}
'Department of Radiation Oncology, Dana-Farber Cancer Institute, Harvard Medical School, Boston, Massachusetts, USA. ${ }^{2}$ Broad Institute of MIT and Harvard, Cambridge, Massachusetts, USA.
${ }^{3}$ Brigham and Women's Hospital, Boston, Massachusetts, USA. ${ }^{4}$ Department of Medical Oncology and ${ }^{5}$ Center for Cancer Cenomics, Dana-Farber Cancer Institute, Boston, Massachusetts, USA. ${ }^{6}$ Danish Cancer Society Research Center, Copenhagen, Denmark. ${ }^{7}$ VA Boston Healthcare System, Harvard Medical School, West Roxbury, Massachusetts, USA. ${ }^{8}$ Division of Hematology and Oncology, Beth Israel Deaconess Medical Center, Boston, Massachusetts, USA
\end{abstract}

Bladder cancer is a genetically heterogeneous disease, and novel therapeutic strategies are needed to expand treatment options and improve clinical outcomes. Here, we identified a unique subset of urothelial tumors with focal amplification of the RAF1 (CRAF) kinase gene. RAF1-amplified tumors had activation of the RAF/MEK/ERK signaling pathway and exhibited a luminal gene expression pattern. Genetic studies demonstrated that RAF1-amplified tumors were dependent upon RAF1 activity for survival, and RAF1-activated cell lines and patient-derived models were sensitive to available and emerging RAF inhibitors as well as combined RAF plus MEK inhibition. Furthermore, we found that bladder tumors with HRAS- or NRAS-activating mutations were dependent on RAF1-mediated signaling and were sensitive to RAF1-targeted therapy. Together, these data identified RAF1 activation as a dependency in a subset making up nearly $20 \%$ of urothelial tumors and suggested that targeting RAF1-mediated signaling represents a rational therapeutic strategy.

Related Commentary: https://doi.org/10.1172/JCI154095

Conflict of interest: J Bellmunt reports stock ownership of Rainier; honoraria from UpToDate; consulting/advising with Pierre Fabre, Astellas, Pfizer, Merck, Genentech (Roche), Novartis, AstraZeneca, MedImmune, and Bristol Myers Squibb; research funding from Millennium, Sanofi, Pfizer, and EMD Serono; and travel reimbursement from Pfizer, MSD Oncology, and Ipsen. DJK reports advising/consulting with Novartis, AADi, and Genentech (Roche) and research support from Genentech (Roche) and Revolution Medicines. GPS reports advising/ consulting with Bristol Myers Squibb, Genentech (Roche), EMD Serono, Merck, Sanofi, Seattle Genetics/Astellas, AstraZeneca, Exelixis, Janssen, Bicycle Therapeutics, Pfizer, and Immunomedics; research support from Sanofi, AstraZeneca, and Immunomedics; travel reimbursement from Bristol Myers Squibb and AstraZeneca; speaking fees from Physicians Education Resource, OncLive, Research to Practice, and Medscape; writing fees from UpToDate and Elsevier (editor of Practice Update Bladder (ancer Center of Excellence); and steering committee membership of trials/studies with Bristol Myers Squibb, Bavarian Nordic, Seattle Cenetics, and QED (all unpaid) and AstraZeneca, EMD Serono, and Debiopharm (all paid). EMVA reports advising/consulting from Tango Therapeutics, Genome Medical, Invitae, Enara Bio, Janssen, Manifold Bio, and Monte Rosa; research support from Novartis and Bristol Myers Squibb; equity in Tango Therapeutics, Genome Medical, Syapse, Enara Bio, Manifold Bio, Microsoft, and Monte Rosa; travel reimbursement from Genentech (Roche); and institutional patents filed on "Cenomic biomarker of response to immunotherapy" (US Patent Application US20170115291A1) and "Methods, systems apparatus, and optimization for effective clinical analysis of cancer genomic data" (US Patent Application W02015013191A1). KWM reports advising/consulting with Pfizer and EMD Serono and research support from Pfizer.

Reference information: J Clin Invest. 2021;131(22):e147849.

https://doi.org/10.1172/JCl147849.

Submitted: February 3, 2021; Accepted: September 16, 2021; Published: November 15 , 2021

Copyright: @ 2021, American Society for Clinical Investigation.

\section{Introduction}

Bladder cancer accounts for approximately 80,000 new cancer diagnoses and nearly 20,000 deaths annually in the United States (1). Approximately $25 \%$ of patients present with muscle-invasive bladder cancer (MIBC), an aggressive but potentially curable disease state. Large-scale genomic studies of MIBC performed by The Cancer Genome Atlas (TCGA) and others have identified numerous recurrent genomic alterations, including mutations, copy number alterations, and chromosomal translocations (2). Additionally, MIBCs exhibit distinct gene expression patterns, and a recent consensus subtyping effort has defined 6 transcriptional subtypes (3).

For several decades, cisplatin-based chemotherapy has been the backbone of systemic therapy for patients with MIBC or metastatic bladder cancer. More recently, anti-PD1/PD-L1 agents have also shown activity in a subset of bladder cancer patients, and multiple agents are now approved in the metastatic setting $(4,5)$. In addition, genomic insights have contributed to the development of targeted agents, such as the fibroblast growth factor receptor (FGFR) inhibitor erdafitinib, which was recently approved for use in advanced bladder cancer patients with tumors harboring activating alterations in FGFR2/3 $(6,7)$. Despite these recent advances, only a subset of patients respond to cisplatin-based chemotherapy or anti-PD/PD-L1 agents, and only $20 \%$ of patients have a tumor FGFR2/3 alteration. Therefore, additional therapeutic strategies are needed.

The MAPK pathway is a 3-tiered signaling cascade comprising the proteins RAF, MEK, and ERK, which are serine/threoninespecific kinases that control critical cellular processes, such as 

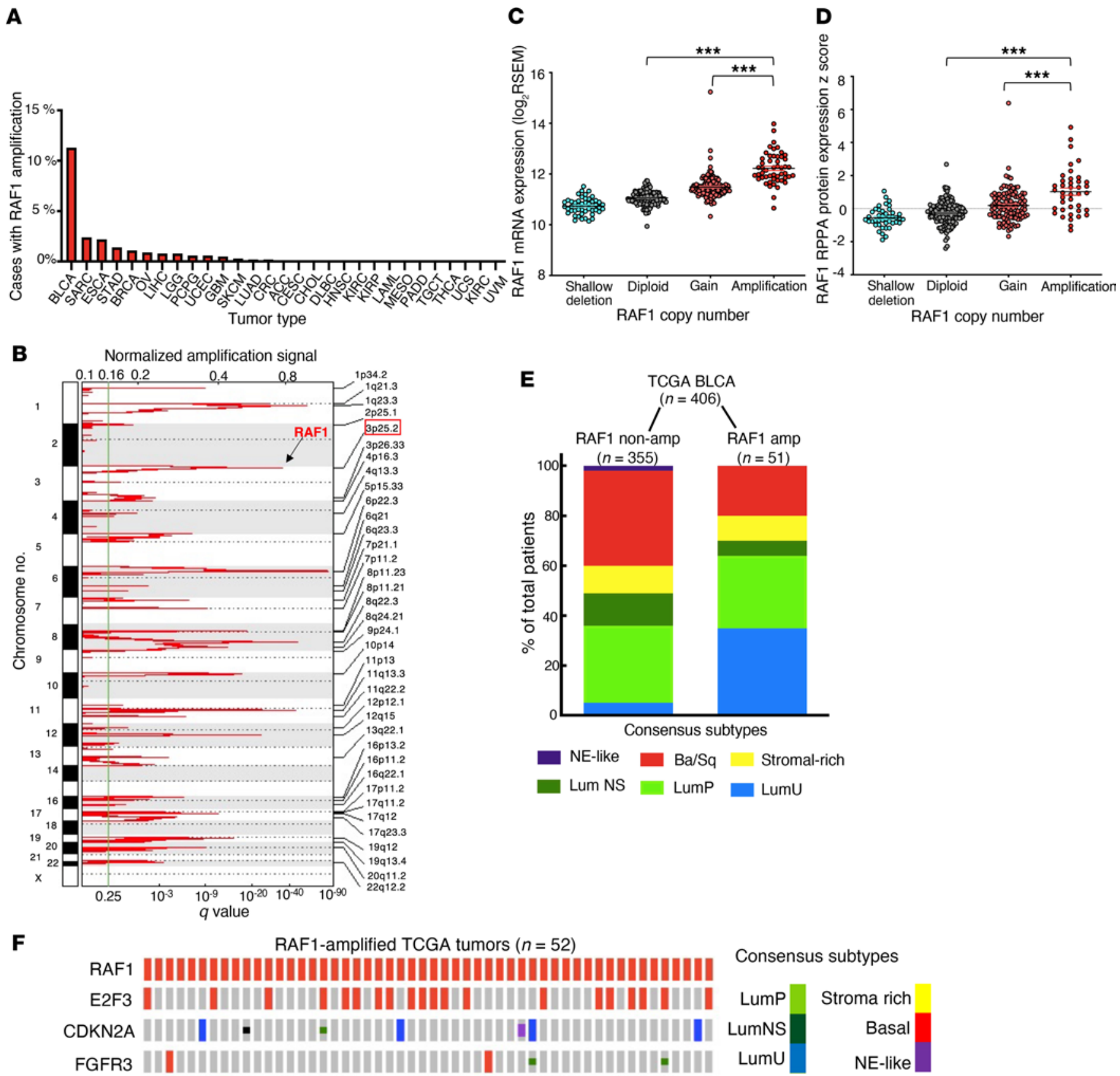

TP53 - "

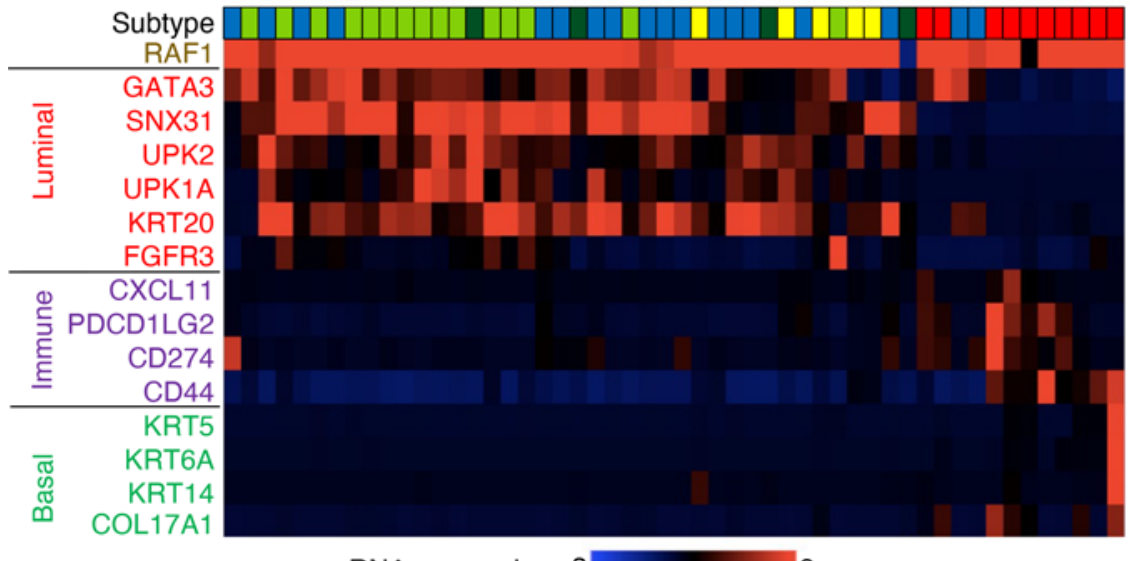

Genetic alterations

No alterations

Amplification

Deep deletion

Truncating mutation

(putative driver)

Missense mutation

(putative driver)

Fusion

mRNA expression -3 
Figure 1. RAF1 is focally amplified in a subset of MIBCs. (A) Frequency of RAF1 amplification across the TCGA pan-cancer cohort. (B) Copy number analysis by GISTIC2 shows recurrent amplifications in the TCGA BLCA cohort. The RAF1 gene is located on chromosome 3p25.2 ( $q=7.6031 \times$ $10^{-36}$ ). (C) RAF1 gene expression by RAF1 copy number status in the TCGA BLCA cohort. (D) RAF1 protein expression z score by RAF1 copy number status in the TCGA BLCA cohort. ${ }^{* *} P<0.001$, ANOVA with Bonferroni's post hoc test. (E) Percentages of RAF1-amplified and RAF1 nonamplified tumors from the TCGA BLCA cohort belonging to each of the 6 consensus transcriptional subtypes. (F) Copy number, mutation status, and mRNA expression of select genes from the RAF1-amplified tumors from the TCGA BLCA cohort $(n=52)$.

differentiation, proliferation, migration, and survival (8-12). RAF is activated by small GTPases of the RAS superfamily, including HRAS, NRAS, and KRAS. Activated RAF (MAP3K) activates MEK (MAP2K), which in turn activates ERK (MAPK; refs.12,13). Humans encode 3 RAF isoforms - ARAF, BRAF, and RAF1 (CRAF) - each located on a different chromosome, but with conserved structural regions, including a RAS-binding domain (RBD), a cysteine-rich membrane recruitment domain (CRD), and a kinase domain (12, 13). RAF activation involves recruitment by activated RAS, release of the RAF N-terminal regulatory subunit through RAS binding, and phosphorylation of key residues, which allow the RAF kinase domain to achieve maximal activity through dimerization-induced allosteric stabilization $(10,14)$.

The RAFs - particularly BRAF and RAF1 - are proto-oncogenes (13), and alterations of RAS/RAF members or activation of upstream receptor tyrosine kinases (such as EGFR or FGFR) occurs commonly in cancer. Among the RAF isoforms, BRAF is the most frequently altered in cancer, with V600-activating mutations occurring in more than half of melanoma and thyroid tumors as well as in smaller percentages of colorectal and other tumor types (14-16). BRAF ${ }^{\mathrm{V} 600 \mathrm{E}}$ can signal as a monomer, whereas RAF1 functions as an obligate homodimer or heterodimer with BRAF (17-20). Inhibitors such as vemurafenib specifically target monomeric V600-mutant $\operatorname{BRAF}(21,22)$, and $\mathrm{BRAF}^{\mathrm{V} 600}$-targeted therapies delivered alone or in combination with a MEK inhibitor are now first-line treatment options in several $\mathrm{BRAF}^{\mathrm{V} 600}$-mutant disease settings $(23,24)$. Newer-generation RAF inhibitors with distinct mechanisms of action, such as inhibition of RAF dimerization, are being developed and have the potential to preferentially target specific RAF isoforms or RAF mutations beyond $\mathrm{BRAF}^{\mathrm{V} 600}$ while also avoiding paradoxical MAPK pathway activation observed with monomeric RAF inhibitors (25-27). The safety and efficacy of several of these agents are currently being tested in patients with tumors harboring MAPK pathway alterations (14).

Here, we identify a subset of MIBCs with focal amplification of $R A F 1$, and we show that $R A F 1$-amplified bladder cancer preclinical models are dependent on $R A F 1$-mediated signaling for survival. Pan-RAF inhibitors as well as newer RAF-directed agents with novel mechanisms of action preferentially target $R A F 1$-amplified bladder cancer cells in vitro and in vivo and are also active in HRAS- and NRAS-mutant models that are dependent on $R A F 1$ activity for survival. Taken together, these data identify a subset of molecularly defined bladder tumors that could be targeted using RAF1-directed agents.

\section{Results}

RAF1 is amplified in a subset of bladder tumors. We compared RAF1 amplification status across tumor types in the TCGA pan-cancer atlas (https://www.cancer.gov/tcga) and found that RAF1 amplification was present in $12 \%$ of bladder tumors (TCGA bladder urothelial carcinoma [BLCA] cohort) but was rare $(<3 \%)$ or absent in other tumor types (ref. 28 and Figure 1A). Analysis of copy-number data from the TCGA BLCA cohort revealed focal amplification of a segment of chromosome $3 p$ harboring the RAF1 gene $\left(q=7.6031 \times 10^{-36}\right.$; Figure 1B). RAF1 amplification was strongly correlated with increased levels of RAF1 mRNA and protein $(P<$ 0.0001; Figure 1, C and D).

Bladder tumors can be classified by gene expression patterns, and a recent consensus MIBC classification scheme defined 6 mRNA-based transcriptional subtypes (3). Although RAF1 amplification is present in all subtypes except neuroendocrine, RAF1 amplification was enriched in the luminal unstable (LumU) subtype (35\% vs. $10 \% ; P \leq 0.0017$, Bonferroni's corrected $t$ test; Figure $1 \mathrm{E}$ and Supplemental Figure 1; supplemental material available online with this article; https://doi.org/10.1172/JCI147849DS1). Accordingly, expression of luminal genes was higher in RAF1amplified tumors compared with nonamplified tumors, whereas expression of immune and basal genes was low in most RAF1amplified tumors (Figure $1 \mathrm{~F}$ and Supplemental Figure 2).

Total mutation count was significantly higher in RAF1-amplified tumors than in nonamplified tumors (median no. of mutations, 294 vs. $166 ; P=3.8 \times 10^{-4}$; Supplemental Figure 3A); however, $R A F 1$ amplification was not significantly correlated with the mutation status of any of the 58 significantly mutated genes in the TCGA BLCA cohort after multiple-hypothesis testing; however, there was a trend toward cooccurrence of RAF1 amplifications with TP53 alterations $(P=0.02)$, consistent with the observed enrichment of TP53 alterations in LumU tumors. RAF1-amplified tumors also had higher levels of genomic instability compared with nonamplified tumors (median fraction genome altered, 0.39 vs. $0.27, P=$ $6.9 \times 10^{-4}$; Supplemental Figure $3 \mathrm{~B}$ ). Among genomic alterations that are particularly relevant in luminal subtypes, $R A F 1$ amplifications cooccurred with E2F3/SOX 4 amplifications $(q=0.0038)$ and amplification of adjacent genes on chromosome $3 \mathrm{p}$, including PPARG $\left(q<10^{-10}\right.$; Supplemental Table 1$)$, but were mutually exclusive with $C D K N 2 A / B$ deletions $(q=0.01$; Figure $1 F)$. $R A F 1$ amplifications were not associated with FGFR3 alterations (either activating S249C mutations or gene amplification), perhaps because FGFR3 alterations are more common in the luminal-papillary (LumP) subtype, whereas RAF1 amplifications are most frequent in the LumU subtype. There was no difference in overall survival of patients with RAF1-amplified versus nonamplified tumors in the TCGA BLCA cohort (Supplemental Figure 3C).

We next analyzed targeted tumor DNA-sequencing data from 472 urothelial cancer cases across grades and anatomic sites from the Dana-Farber Cancer Institute/Brigham and Women's Cancer Center (29). Upon manual review of copy-number data, we identified 54 cases (11\%) with 5 or more copies of RAF1 (Supplemental Table 2, Figure 2A, and Supplemental Figures 4 and $5)$. Although the cohort included both high-grade $(n=380)$ and low-grade $(n=92)$ cases, all RAF1-amplified tumors were high grade. For a subset of these cases, we used available formalin-fixed 
A
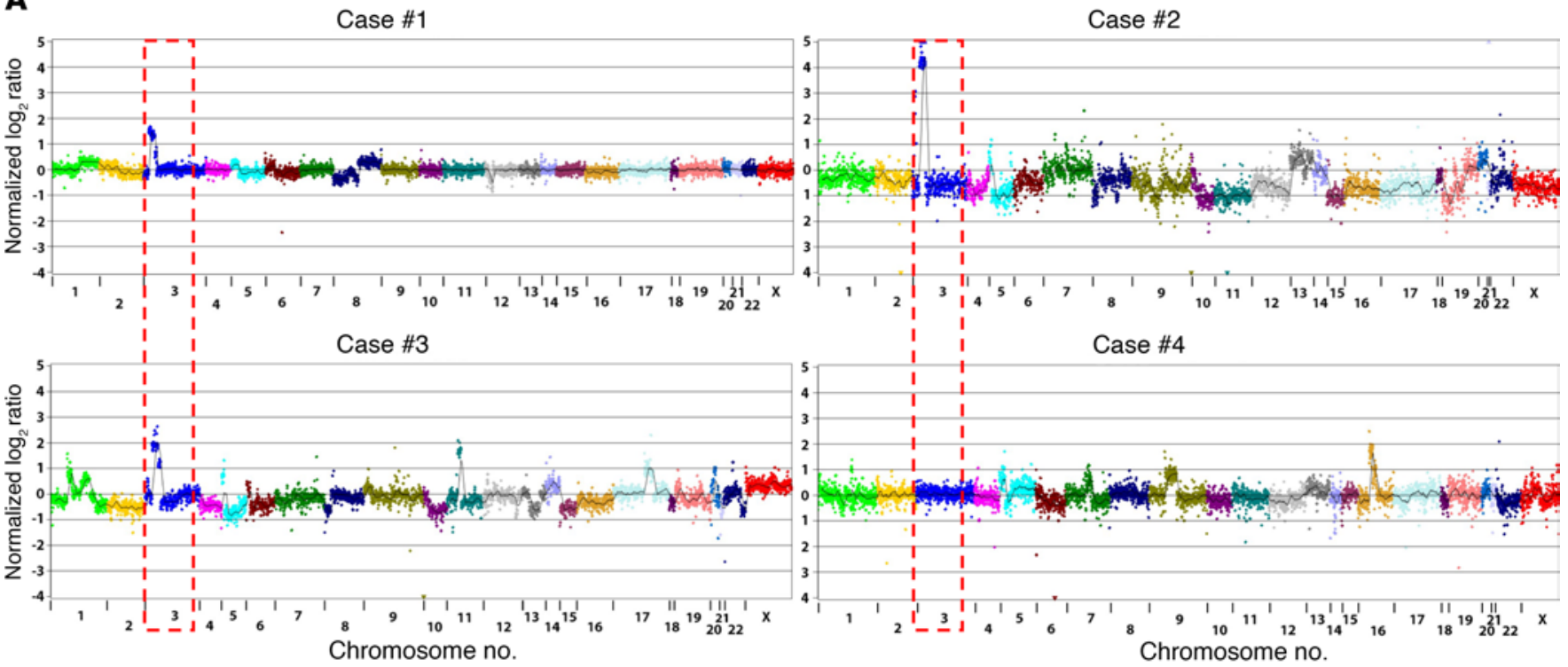

B

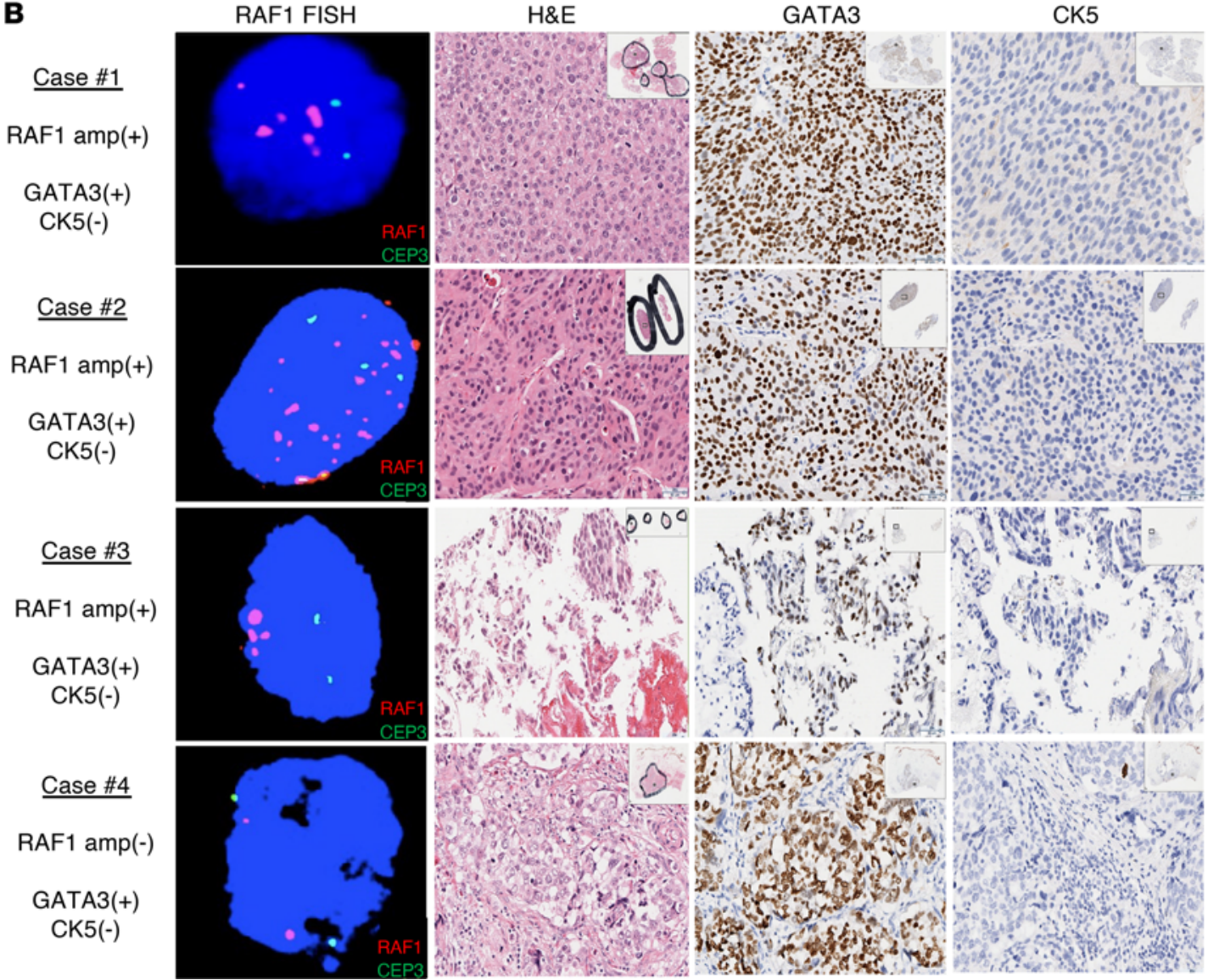

Figure 2. Focal amplification and luminal differentiation in RAF1-amplified bladder tumors. Representative RAF1-amplified (cases 1-3) and RAF1 nonamplified (case 4) bladder tumors from the Dana-Farber Cancer Institute/Brigham and Women's Cancer Center. (A) For the RAF1-amplified cases, copy number analysis from targeted next-generation sequencing shows focal amplification of the RAF1 locus on chromosome 3 (denoted by red hatched box). (B) FISH analysis using a RAF1-specific probe (red) shows more than 2 RAF1 foci per cell (chromosome 3 centromeric probe [CEP3] shown in green). Tumor $\mathrm{H} \& \mathrm{E}$ and immunohistochemical staining for the luminal marker CATA3 and basal marker CK5 show a staining pattern consistent with luminal differentiation in RAF1-amplified tumors. Original magnification, $\times 20$; insets, $\times 1$ (unmagnified). 
paraffin-embedded (FFPE) tumor tissue to perform FISH with a $R A F 1$-specific probe (Figure 2B). Tumors with RAF1 amplification identified by tumor sequencing had more than $2 R A F 1$ foci per nucleus in all or nearly all tumor cells (Supplemental Figure 6A), consistent with clonal amplification of the RAF1 locus in tumor cells. $\mathrm{H} \& \mathrm{E}$ and IHC staining of the tumors revealed that RAF1-amplified tumors were positive for the luminal marker GATA3 and negative for the basal marker cytokeratin 5 (CK5), consistent with a luminal phenotype (Figure 2B, Supplemental Figure 6B, and ref. 30)

RAF1-amplified bladder tumors are dependent on RAF1 signaling for survival. To investigate the functional role of $R A F 1$ in bladder cancer, we first sought to identify bladder cancer cell lines with $R A F 1$ amplification. We examined available RAF1 copy number and mRNA levels across 36 bladder cancer cell lines from the Broad Institute Cancer Dependency Map (DepMap) (https://depmap. org/portal/depmap/) $(31,32)$ and identified 2 bladder cancer cell lines - 5637 and UMUC9 - that had RAF1 copy number amplification and a corresponding increase in RAF1 mRNA expression (Figure 3A). To confirm RAF1 amplification in these lines, we performed RAF1 immunoblotting across a panel of bladder epithelial and tumor cell lines and observed highest $R A F 1$ protein expression levels in 5637 and UMUC9 (Figure 3B).

To determine whether RAF1-amplified cell lines are dependent on $R A F 1$ for survival, we depleted $R A F 1$ using siRNAs. We observed significantly increased sensitivity to RAF1 depletion in RAF1-amplified compared with nonamplified bladder cancer cell lines (Figure 3, C and D, and Supplemental Figure 7). Furthermore, RAF1 depletion resulted in decreased activity of RAF/MEK/ERK signaling in RAF1-amplified cell lines (Figure 3E), whereas no change in PI3K/ AKT pathway signaling was observed following RAF1 depletion (Supplemental Figure 8). These data indicate that RAF1-amplified bladder tumors are dependent on RAF1-mediated signaling for survival. We further confirmed the essentiality of RAF1 in RAF1-amplified cell lines by analyzing gene-dependency scores from published CRISPR/Cas9 screening data (33). RAF1-amplified cell lines were among the cell lines that were most dependent on $R A F 1$ for survival (Figure 3F and Supplemental Table 3). Other dependent cell lines harbored alternate mechanisms of MAPK pathway activation, such as an activating NRAS or HRAS mutation, as discussed below.

RAF1-amplified cells are sensitive to RAF and RAF plus MEK inhibition. Given the sensitivity of RAF1-amplified bladder cancer cells to RAF1 depletion, we wished to determine whether RAF1amplified tumors are also sensitive to pharmacologic inhibition of RAF1-mediated signaling. We extracted drug-sensitivity data from the DepMap for all 14 available bladder cancer cell lines treated with either the pan-RAF inhibitor RAF265 (34) or the BRAFv600E inhibitor PLX4720 (ref. 35 and Figure 4A), which were the only RAF inhibitors available in the data set. This data set includes the RAF1-amplified cell line 5637 but does not include UMUC9. Although neither RAF265 nor PLX4720 specifically targets RAF1, the $R A F 1$-amplified cell line 5637 is among the most sensitive cell lines to each of these 2 agents, suggesting increased sensitivity to inhibition of RAF-mediated signaling. Other sensitive cell lines harbor alternative mechanisms of MAPK pathway activation, including HRAS or NRAS mutations.

To further investigate sensitivity to pan-RAF inhibition, we measured cell viability of several cell lines following treatment with RAF265. The RAF1-amplified 5637 and UMUC9 cell lines demonstrated significantly higher sensitivity to RAF265 than the nonamplified J82 cell line (Figure 4B). Ku-19-19 is a bladder cancer cell line with an activating NRAS Q61R mutation, which we hypothesized may also confer increased sensitivity to RAF265. Indeed, although activating NRAS mutations are rare in bladder cancer (1\% frequency in TCGA BLCA cohort), the NRAS-mutant Ku-19-19 cell line demonstrated sensitivity to RAF265 similar to that of RAF1-amplified cell lines (Figure 4B).

Preclinical and clinical data support the use of combination approaches that simultaneously target BRAF and MEK in BRAF ${ }^{\mathrm{V} 600 \mathrm{E}}$ mutant tumor settings $(23,36)$. To investigate the activity of combined RAF and MEK inhibition in RAF1-amplified bladder cancer, we measured survival of UMUC9 (RAF1-amplified) and J82 (RAF1 nonamplified) cell lines following treatment with the pan-RAF inhibitor RAF265 and the MEK inhibitor trametinib (Figure 4C). J82 was minimally sensitive to RAF265 and trametinib alone or in combination, whereas UMUC9 showed sensitivity to each agent alone and increased sensitivity to the combination. Similarly, in a 10-day colony-formation assay, RAF1-amplified UMUC9 and 5637 cell lines were significantly more sensitive to the combination of RAF265 and trametinib than to RAF265 alone (Figure 4D). Consistent with increased sensitivity to combined RAF plus MEK inhibition, we observed more complete inhibition of RAF/MEK/ERK signaling with RAF265 plus trametinib treatment compared with RAF265 treatment alone (Figure 4E).

Cisplatin-based chemotherapy is a common treatment for advanced bladder cancer, and we therefore sought to characterize the interaction between cisplatin and RAF inhibition. We performed a series of cell-viability assays using combinations of cisplatin and RAF265 in the RAF1-amplified cell lines UMUC9 and 5637. Overall, we observed a pattern of additive activity with mild synergy observed at low cisplatin concentrations in the 5637 cell line. Importantly, there was no evidence of antagonism (Supplemental Figure 9).

$R A F$ and RAF plus MEK inhibition is active in RAF1-amplified tumors in vivo. We next tested the activity of RAF inhibition alone or in combination with MEK inhibition in RAF1-amplified tumors in vivo. RAF265 was developed as an inhibitor of mutant BRAF but also potently inhibits WT BRAF, RAF1, and other kinases (34). In preclinical studies, RAF265 demonstrated antitumor activity in $B R A F$-mutant and WT BRAF tumor models, and responses were also observed in both $B R A F$-mutant and WT BRAF metastatic melanoma patients in a phase I clinical trial $(37,38)$. Therefore, we elected to test the activity of RAF265 in RAF1-amplified bladder tumor xenografts. We implanted RAF1-amplified UMUC9 cells in immunodeficient mice and randomized mice to treatment with vehicle or with RAF265 alone or in combination with the MEK inhibitor trametinib. Mice were treated twice weekly to a maximum of 9 doses, and mouse weights and tumor measurements were monitored for a total of 39 days following implantation. RAF265 was well tolerated at the tested dose and schedules (Supplemental Figure 10), and we observed significant tumor-growth delay with $30 \mathrm{mg} / \mathrm{kg}$ RAF265 alone and an even more pronounced response when RAF265 was combined with $1 \mathrm{mg} / \mathrm{kg}$ trametinib (Figure 5A). At the conclusion of the experiment, mice were sacrificed and tumors were excised, weighed, and photographed. Tumors treated 
A

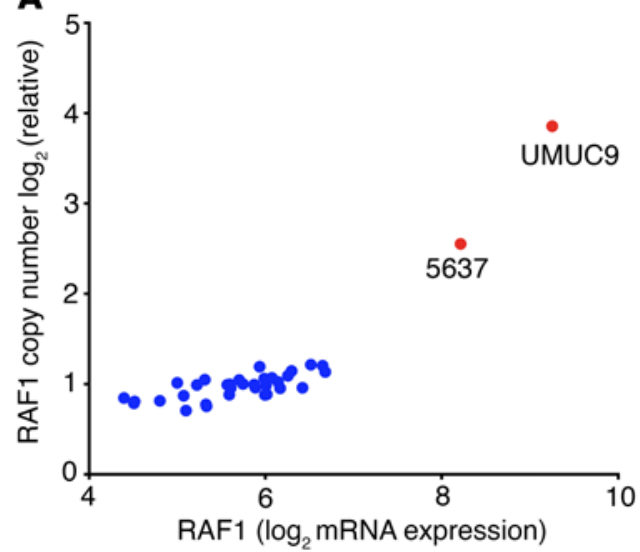

B

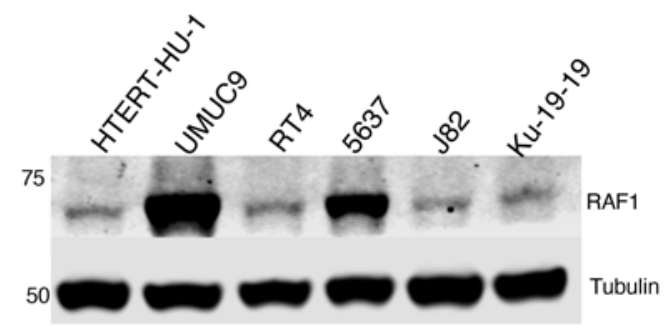

C

5637 (RAF1 amp)

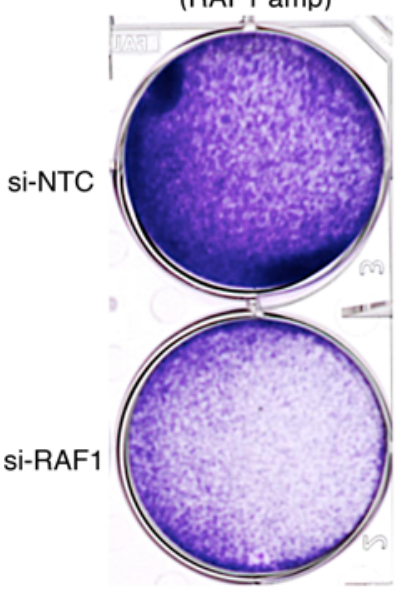

UMUC9 (RAF1 amp)

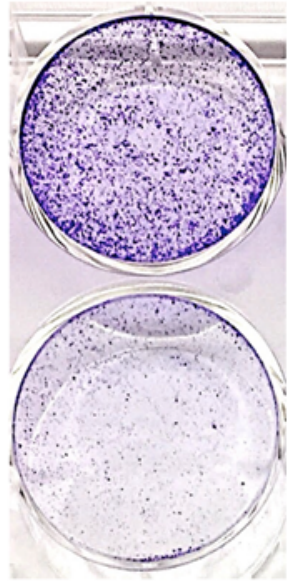

J82 (RAF1 non-amp)

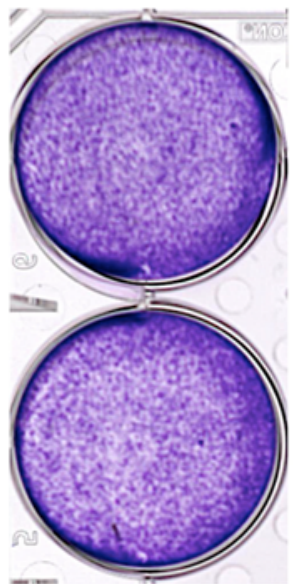

D

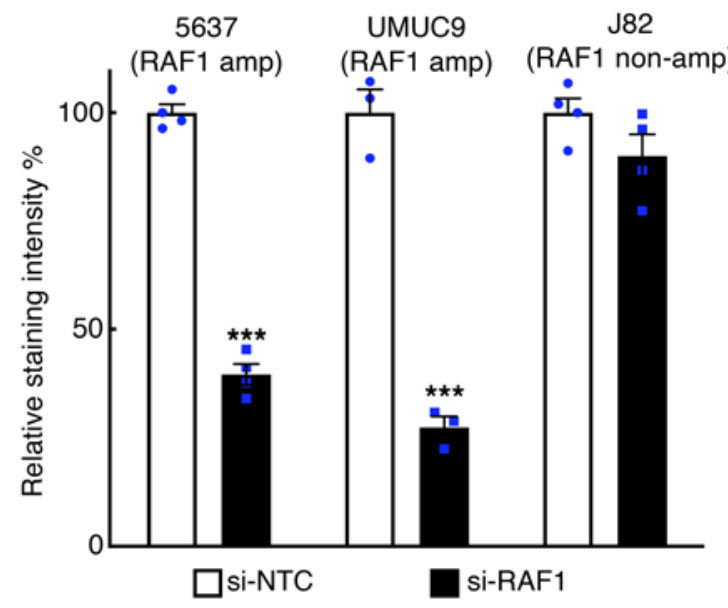

E

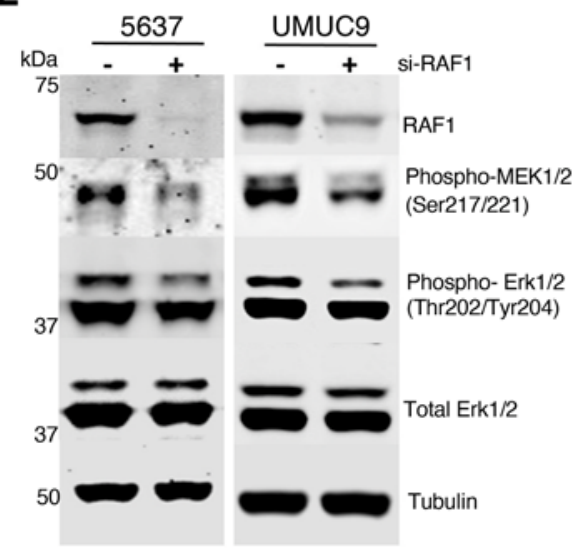

$\mathbf{F}$

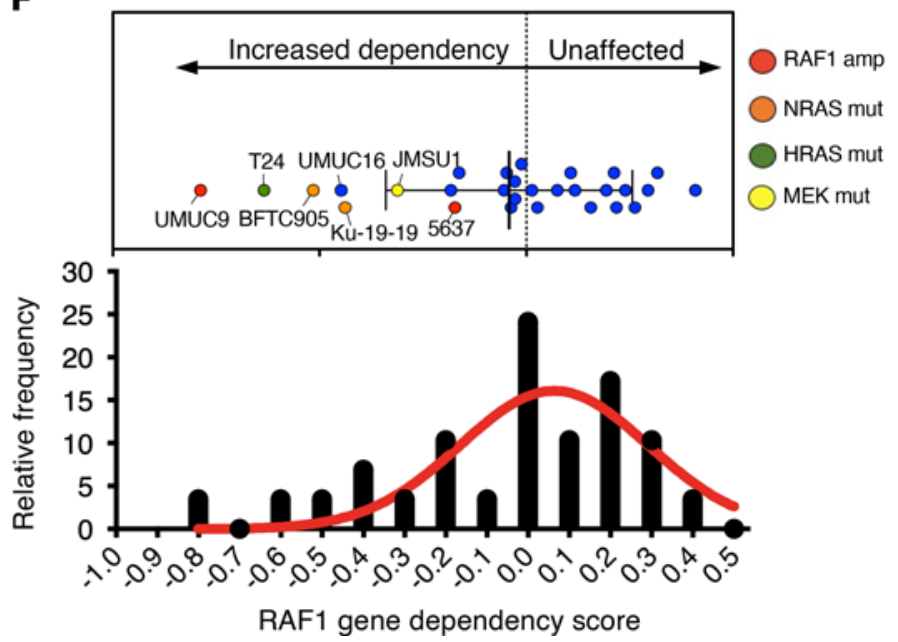

Figure 3. RAF1-amplified cell lines are dependent on RAF1 signaling. (A) RAF1 mRNA expression ( $x$ axis) and DNA copy number ( $y$ axis) across 36 bladder cancer cell lines from the DepMap identify 2 cell lines (5637 and UMUC9) with high RAF1 levels. (B) RAF1 immunoblot in bladder epithelial and tumor cell lines confirms high levels of RAF1 protein expression in 5637 and UMUC9. (C) RAF1 depletion by siRNA kills RAF1-amplified cell lines, but has minimal effect on bladder cancer cell lines without RAF1 amplification. Unmagnified. NTC, nontargeting control siRNA. (D) Quantification of the relative signal intensity from the viability assay in $\mathbf{C}$. ${ }^{* *} P<0.0001$, ANOVA with Bonferroni's post hoc test. (E) RAF1 depletion by siRNA abrogates RAF/MEK/ERK signaling in RAF1-amplified bladder cancer cell lines, as shown by immunoblot (blots were run in parallel from the same sample). (F) RAF1 gene-dependency scores for bladder cancer cell lines from CRISPR-Cas9 essentiality screens from DepMap (33). A low score indicates a higher likelihood that the gene is essential in a given cell line. RAF1-amplified cell lines (UMUC and 5637) are shown in red, an HRAS mutant cell line (T24) in green, NRAS mutant cell lines (Ku-19-19 and BFTC905) in orange, and a MEK2 mutant cell line (JMSU1) in yellow. Cell lines without alterations in any of these 4 genes are shown in blue. The bottom panel shows the distribution of RAF1 dependency scores across the 29 bladder cancer cell lines analyzed. 
with RAF265 alone or in combination with trametinib weighed significantly less than vehicle-treated tumors (Figure 5, B and C). We performed FISH analysis and RAF1 immunohistochemistry from an untreated UMUC9 xenograft to confirm RAF1 amplification (Figure 5D and Supplemental Figure 11).

To further validate $R A F 1$ amplification as a therapeutic target in bladder cancer, we leveraged a patient-derived xenograft (PDX) tumor model harboring RAF1 amplification (ref. 39 and Supplemental Figure 12, A and B). Low-passage PDX tumors were implanted in immunodeficient mice, and tumor-bearing mice were subsequently randomized to RAF265 plus trametinib or to no treatment when tumors reached $100 \mathrm{~mm}^{3}$ or larger. Analysis of engrafted tumors showed that the PDX model exhibited luminal features, including positive IHC staining for the luminal marker GATA3 and negative staining for the basal marker CK5 (Figure 6A), similarly to RAF1amplified tumors from TCGA BLCA and our institutional cohort (Supplemental Figure 6). The PDX model also exhibited a RAF1-staining pattern that resembled the staining pattern of tumors with highest RAF1 expression from the Human Protein Atlas (40). Mice in the treatment group received $30 \mathrm{mg} / \mathrm{kg}$ RAF265 plus $1 \mathrm{mg} / \mathrm{kg}$ trametinib delivered i.p. twice weekly. Tumor growth was monitored by serial volumetric analyses, and tumors in the treatment group were significantly smaller than tumors in the untreated group (Figure 6B and Supplemental Figure 12C). Mice in the untreated group had a median survival of 24 days, and all were sacrificed when they met the protocol-defined morbidity end point, whereas treated mice remained healthy and were sacrificed at the end of the experiment $(P=0.0008$; Figure $6 C)$. Tumors from treated mice were significantly smaller than the tumors from untreated mice (average weight $1.6 \mathrm{~g}$ vs. $0.34 \mathrm{~g}, P=0.0008$; Figure $6, \mathrm{D}$ and $\mathrm{E}$ ) and had increased IHC staining of cleaved PARP and $\gamma \mathrm{H} 2 \mathrm{AX}$ as well as decreased staining of Ki-67 (Supplemental Figure 13), consistent with increased apoptosis and decreased proliferation induced by RAF265 plus trametinib treatment. Together, these data demonstrate activity of combined RAF plus MEK inhibition in a patient-derived RAF1-amplified tumor model.

RAF and RAF plus MEK inhibition is also active in NRAS and HRAS mutant bladder tumors. Recent biochemical and cell-based studies have identified distinct binding preferences of RAS proteins to their downstream RAF targets (41). Although RAF1 binds to all RAS members with high affinity, HRAS was found to bind preferentially to RAF1 over BRAF. In bladder cancer, HRAS mutations are more common than mutations in either KRAS or NRAS. Approximately $4 \%$ of tumors in the TCGA BLCA cohort harbor an activating HRAS mutation, and the observed frequency is $12 \%$ in tumors arising in the ureter or renal pelvis $(42-44)$. In our institutional urothelial tumor cohort, activating HRAS mutations were present in $4 \%$ of tumors across the cohort, but occurred in $13 \%$ of upper tract tumors (Supplemental Table 4). In both cohorts, HRAS mutations and RAF1 amplification were mutually exclusive.

Given the preference of HRAS binding to RAF1, we hypothesized that RAF1 or combined RAF1 plus MEK inhibition may preferentially target bladder tumors with activating HRAS or NRAS mutations. The T24 cell line harbors an HRAS G12V-activating mutation, whereas the Ku-19-19 and BFTC905 cell lines have an NRAS Q61L-activating mutation. Analysis of CRISPR-Cas9 essentiality screening data demonstrated that these lines were selectively dependent upon their respective mutant RAS for survival (Figure 7A, Supplemental Table 5, and ref. 33) Furthermore, these HRAS and NRAS mutant cell lines were also among the most dependent on $R A F 1$ for survival (Figure 3F), consistent with mutant RASinduced $R A F 1$ activation.

In order to determine whether RAF1 inhibition represents a therapeutic strategy for HRAS and NRAS mutant bladder tumors, we tested the activity of RAF inhibitors in T24 (HRAS mutant) and Ku-19-19 (NRAS mutant) cell lines. Similarly to the RAF1amplified UMUC9 and 5637 cell lines, both HRAS-mutant T24 and NRAS-mutant $\mathrm{Ku}-19-19$ cell lines were highly sensitive to RAF265 alone or in combination with trametinib (Figure 7B). LHX254 is a potent and selective WT BRAF and RAF1 inhibitor that has activity in RAS-driven preclinical models and is currently being investigated in numerous clinical trials in patients with MAPK-altered tumors $(45,46)$. LXH254 potently inhibited ERK activation (Figure 7C), leading to apoptotic cell death as measured by increased caspase activity, cleaved PARP, and $\gamma \mathrm{H} 2 \mathrm{AX}$ levels in $H R A S$ - and NRAS-mutant cell lines as well as in the RAF1-amplified cell lines (Supplemental Figures 14-16).

Given the proven clinical activity of combining BRAF plus MEK inhibition in $\mathrm{BRAF}^{\mathrm{V} 600}$-mutant tumor settings, we reasoned that combining a potent RAF inhibitor such as LHX254 with a MEK inhibitor such as trametinib may represent the most promising therapeutic strategy for targeting RAF1-amplified or HRAS/ NRAS-mutant bladder tumors. We first investigated the combination activity of LHX254 and trametinib in vitro and observed additive effects on cell viability with modest synergy noted at low LHX254 concentrations (Supplemental Figure 17). Finally, we tested the activity of the RAF inhibitors RAF265 and LXH254 alone or in combination with trametinib in mice bearing NRAS mutant Ku-19-19 xenografts. Mice were treated twice weekly for 2 weeks, and tumor volumes were monitored for a total of 14 days following the first dose. NRAS mutant Ku-19-19 xenografts grew rapidly in untreated mice, whereas treated mice had significant tumor growth delay and reduction in tumor volume following initiation of treatment with either LXH254 (30 mg/ $\mathrm{kg}$ ) alone, trametinib alone, LXH254 plus trametinib, or RAF265 plus trametinib (Figure 7D and Supplemental Figure 18). At the conclusion of the experiment, mice were sacrificed and tumors were excised, weighed, and photographed. The average weight of tumors treated with LXH254 or LXH254/RAF265 plus trametinib was significantly lower than in vehicle-treated mice (Figure 7, E and F).

\section{Discussion}

For decades, cytotoxic chemotherapy was the only systemic therapy with proven efficacy in urothelial cancer. In the past several years, large-scale genomic analyses have elucidated the molecular features of bladder cancer and are informing the search for targeted therapies. Indeed, insights gained from genomic studies contributed to the development and recent approval of the FGFR inhibitor erdafitinib for treatment of bladder cancer patients with tumor FGFR2/3 alterations $(6,7)$. However, FGFR alterations are only present in a subset of tumors, and identifying novel therapeutic strategies for urothelial tumors remains a pressing clinical need.

In this study, we identify and characterize a subset of urothelial tumors with an amplification of RAF1 (CRAF). While RAF1 

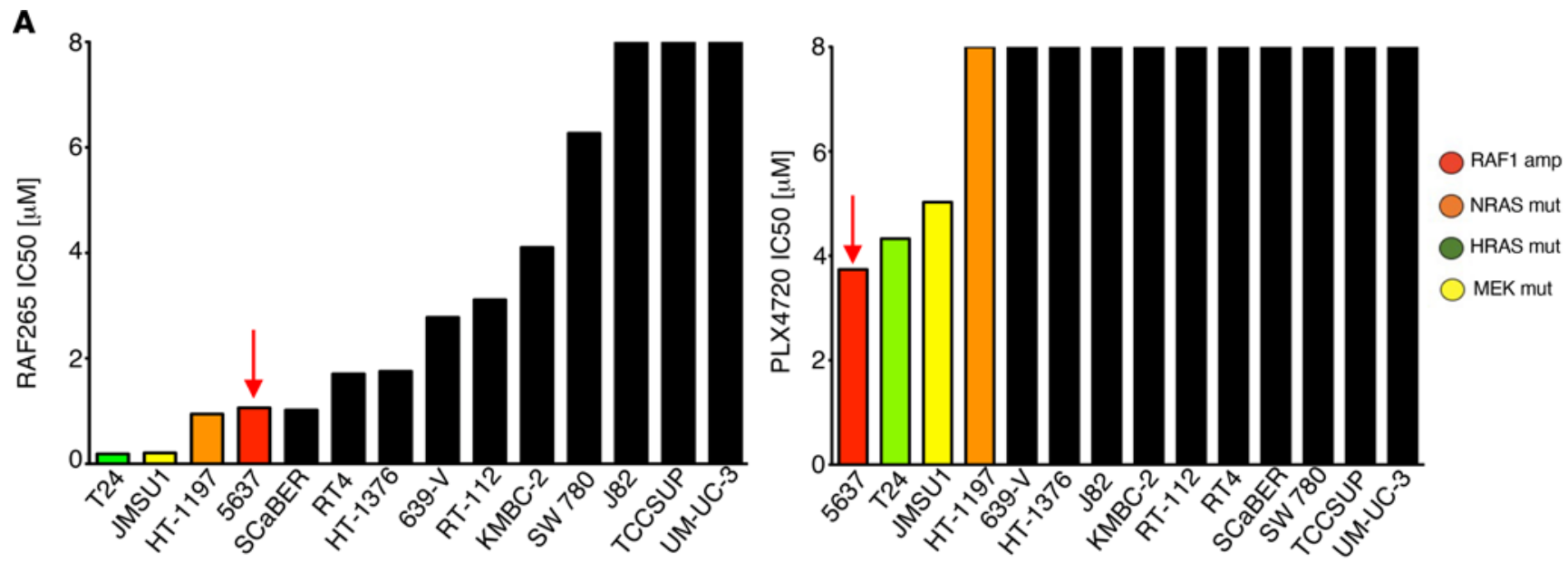
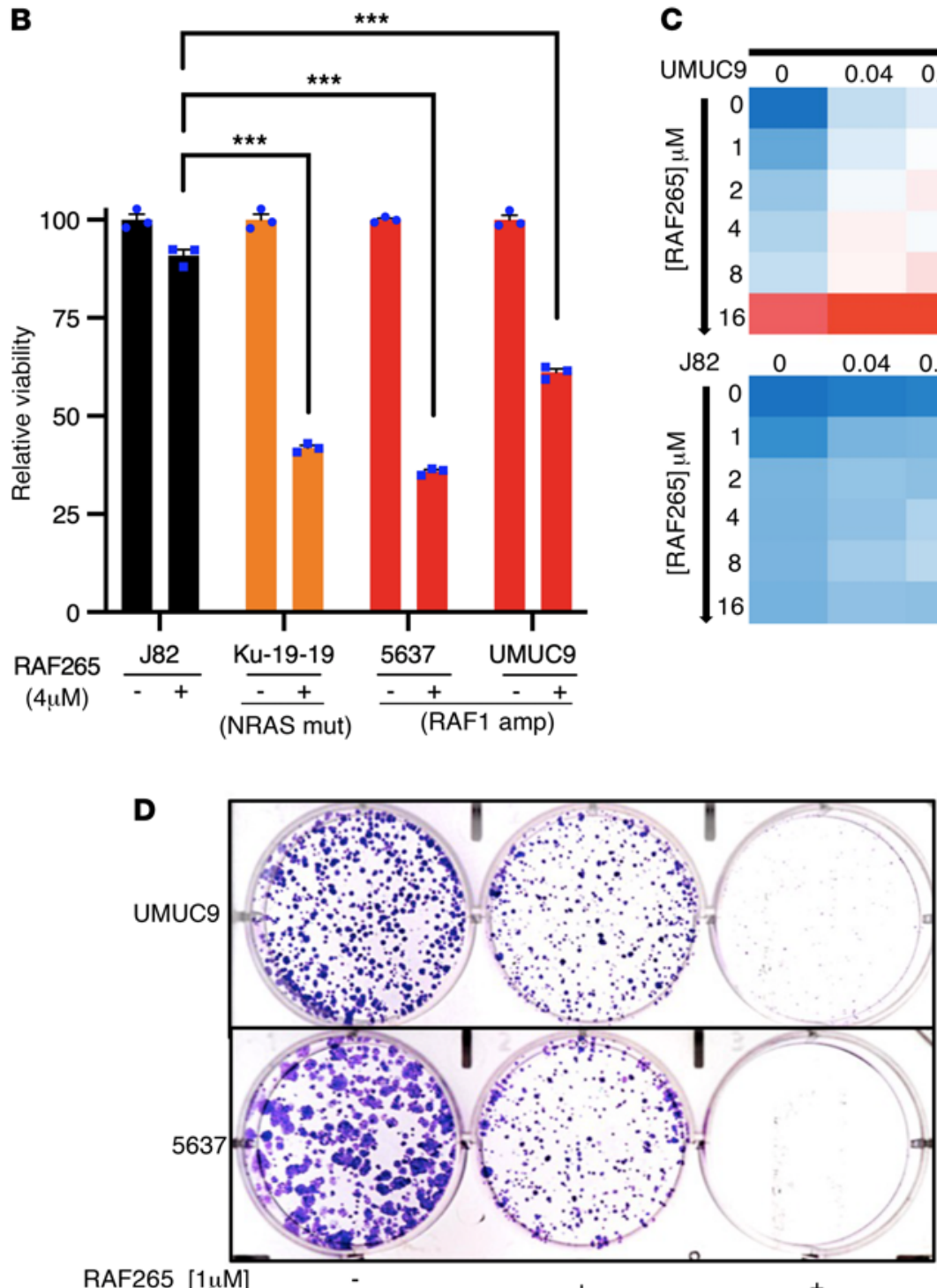

RAF265 $[1 \mu \mathrm{M}]$

Trametinib $[0.5 \mu \mathrm{M}]$
C
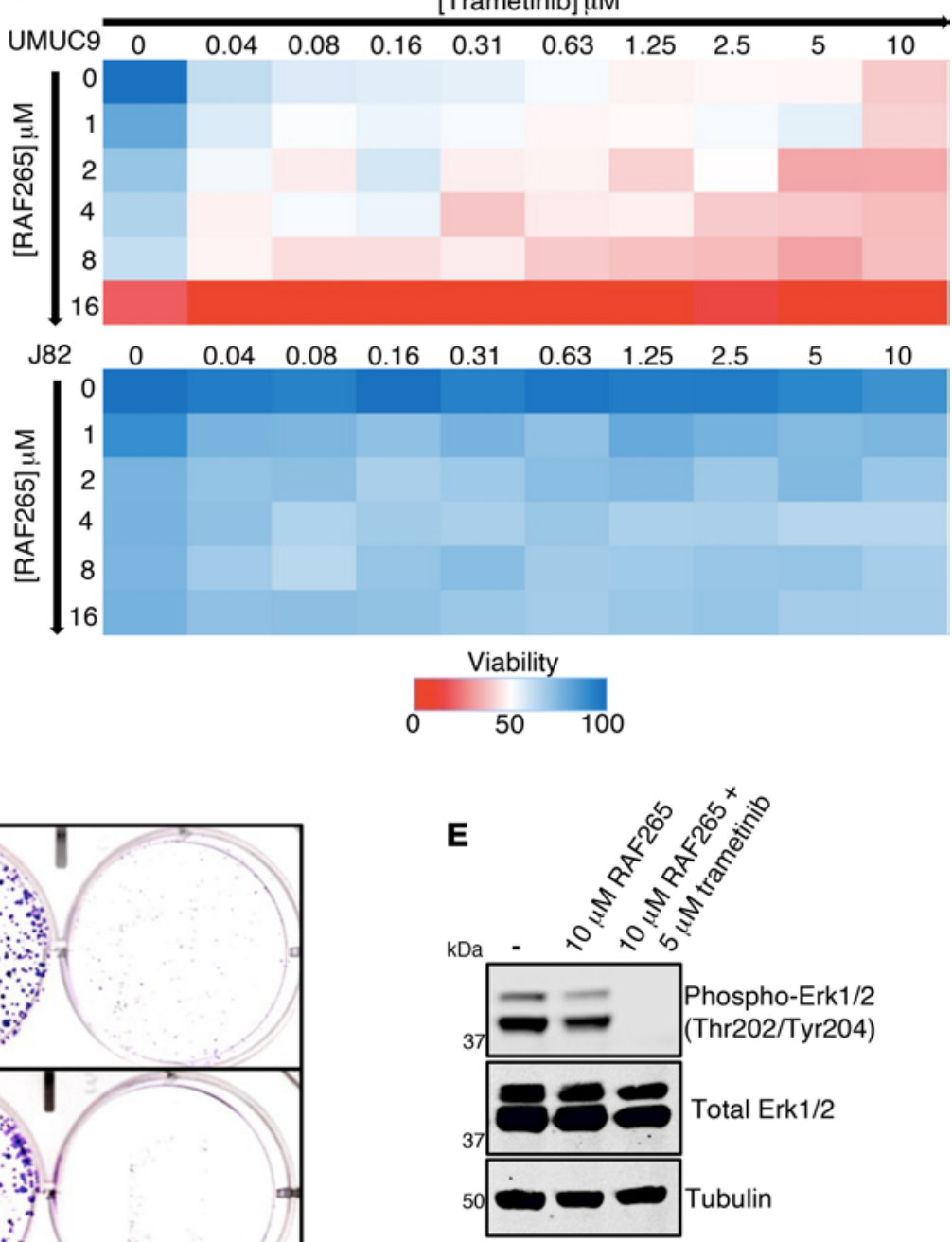
Figure 4. RAF1-amplified cell lines are sensitive to RAF and MEK inhibition. (A) IC $\mathrm{C}_{50}$ values for bladder cancer cell lines from the DepMap data set treated with the pan-RAF inhibitor RAF265 (left) or the BRAFV600E inhibitor PLX4720 (right). The RAF1-amplified bladder cancer cell line (5637) is denoted by red arrows. T24 (green) has an HRAS mutation, JMSU1 (yellow) has a MEK2 mutation, and HT-1197 has an NRAS mutation. Sensitivity data for the RAF1-amplified UMUC9 line were not available. (B) Relative cell viability measured by luminescence assay following 3-day treatment with $4 \mu$ M RAF265. ${ }^{* *} P<0.0001$, ANOVA with Bonferroni's post hoc test. (C) Heatmap showing viability of UMUC9 (RAF1 amplified) and $\int 82$ (RAF1 nonamplified) cells following 3-day treatment with combinations of the pan-RAF inhibitor RAF265 and the MEK inhibitor trametinib. (D) Unmagnified colony-formation assays following treatment of RAF1amplified cell lines (UMUC9 and 5637) with RAF265 and trametinib show increased sensitivity to combination treatment. (E) Immunoblot shows complete ERK inhibition following treatment with the combination of RAF265 and trametinib in UMUC9 cells.

amplifications are very rare in most other tumor types, more than $10 \%$ of bladder tumors in TCGA BLCA and our institutional cohort have amplification of RAF1 $(2,29)$. RAF1 amplifications have also been observed in large bladder tumor cohorts using FISH (47). We find that RAF1 amplifications are accompanied by high levels of RAF1 mRNA and protein as well as by activation of the MAPK (RAF/MEK/ERK) signaling pathway. Although the MAPK pathway is one of the most frequently altered pathways in cancer and can occur through a variety of mechanisms, RAF1 amplification appears to be a mechanism unique to bladder cancer.

$R A F 1$ is nearly universally coamplified with $P P A R G$, which is located adjacent to RAF1 on chromosome $3 p$ and encodes a transcription factor that is a key driver of urothelial luminal differentiation (48). Accordingly, we found that the majority of RAF1amplified tumors express protein markers of luminal differentiation such as GATA3 and belong to one of the luminal transcriptional subtypes, particularly the LumU subtype (Supplemental Figure 19). Activation of PPAR $\gamma$ signaling via PPARG amplification or activating mutation has been shown to contribute to the bladder tumor phenotype and can be pharmacologically targeted by PPARG inverse agonists (49). Interestingly, MAPK signaling through RAF1 has been shown to activate PPARG via phosphorylation of the PPARG coactivator PPAR-binding protein (PBP) (50), suggesting a possible mechanism through which coamplification of RAF1 and PPARG could cooperate to drive luminal bladder tumor growth and raising the possibility of cotargeting RAF1 and PPARG signaling as a therapeutic strategy in this subset of bladder tumors.

Using publicly available data, we identified bladder cancer cell lines $(31,32)$ and a PDX model (39) with RAF1 amplification. Depleting $R A F 1$ in $R A F 1$-amplified cell lines dramatically reduced cell viability, whereas $R A F 1$ depletion had minimal impact in $R A F 1$ nonamplified cell lines. The dependence of $R A F 1$-amplified cell lines on RAF1 is further supported by analysis of large-scale CRISPR screening data (33), which show that RAF1-amplified cell lines are among the most dependent on $R A F 1$ for survival. Interestingly, other cell lines that were also highly dependent on $R A F 1$ harbored other alterations in the MAPK pathway, including activating mutations in HRAS or NRAS. Although RAF1 has high binding affinity for all Ras proteins, RAF1 has been shown to be particularly important for downstream transmission of HRAS-mediated signaling (41). HRAS mutations occur in $3 \%$ to $5 \%$ of urothelial bladder tumors, but are present in $12 \%$ of upper tract tumors (42-44). These genetic data support a critical biological role for RAF1-mediated signaling in RAF1-amplified and HRAS-mutant urothelial tumors and suggest that targeting RAF1-mediated signaling may be a rational therapeutic strategy for this subset that comprises up to $20 \%$ of urothelial tumors.

Activating $\mathrm{BRAF}^{\mathrm{V} 600 \mathrm{E}}$ mutations are common in several tumor types, and the development and clinical implementation of $\mathrm{BRAF}^{\mathrm{V} 600 \mathrm{E}}$ inhibitors has been one of the most successful applications of targeted cancer therapy to date. Nevertheless, BRAF mutations are quite rare in bladder cancers; however, more than $80 \%$ of canine invasive bladder tumors have an activating BRAF V595E mutation (homologous to the V600E mutation in humans; ref. 51). Given our finding that RAF1 amplification is a driver in a subset of human bladder tumors, these data suggest that RAF-mediated signaling is a shared oncogenic driver of bladder tumors across species.

Unlike WT RAFs, which are activated through dimerization, $\mathrm{BRAF}^{\mathrm{V} 600 \mathrm{E}}$ can function as a monomer, and approved BRAF ${ }^{\mathrm{V} 600 \mathrm{E}}$ inhibitors preferentially bind and inhibit monomeric RAFs $(11,14)$. Therefore, targeting tumors with MAPK pathway activation driven by nonBRAF ${ }^{\mathrm{V} 600 \mathrm{E}}$ alterations - including RAF1 amplification requires an alternative strategy, and numerous RAF inhibitors with distinct mechanisms of action are now being tested in patients with tumor MAPK pathway alterations, including those with KRAS or NRAS mutations (Supplemental Table 6 and refs. 46, 52-57).

Using available cell line drug sensitivity data, we found that $R A F 1$-amplified bladder cancer cell lines were sensitive to the pan-RAF kinase inhibitor RAF265, which binds not only RAF1, but also WT BRAF, BRAF ${ }^{\mathrm{V} 600 \mathrm{E}}$, and other intracellular kinases (34, 58). RAF1-amplified cell lines and a RAF1-amplified PDX model were sensitive to RAF265 in vitro and in vivo, and this sensitivity could be enhanced by cotreatment with the MEK inhibitor trametinib. Combining MEK inhibition with BRAF inhibition has been shown to improve response rates, prevent paradoxical ERK activation, and delay onset of resistance $(36,59)$. Similar strategies of combining RAF1 and MEK inhibition may therefore also be necessary to optimally target RAF1 amplification in bladder cancer. Importantly, newer RAF dimerization inhibitors with increased activity and specificity compared with RAF265 are also being developed. One such inhibitor, LHX254 (now naporafenib), potently inhibits WT BRAF and RAF1 while having lower affinity for ARAF (45), and we observed single-agent activity of LHX254 in RAF1-amplified as well as HRAS- and NRAS-mutant bladder cancer models abolishing downstream ERK phosphorylation and inducing apoptosis. Hence, the most promising therapeutic strategies may involve combining newer, selective RAF inhibitors such as LHX254 with MEK/ERK inhibition.

The treatment paradigm for advanced urothelial cancer is rapidly evolving. Eligible patients typically receive cisplatin-based chemotherapy; however, chemotherapy response rates vary across patients, and retrospective clinical-genomic studies suggest that patients with luminal tumors may derive less benefit from chemotherapy than those with basal or neuroendocrine subtypes (60). Given that the majority of $R A F 1$-amplified tumors belong to 
A

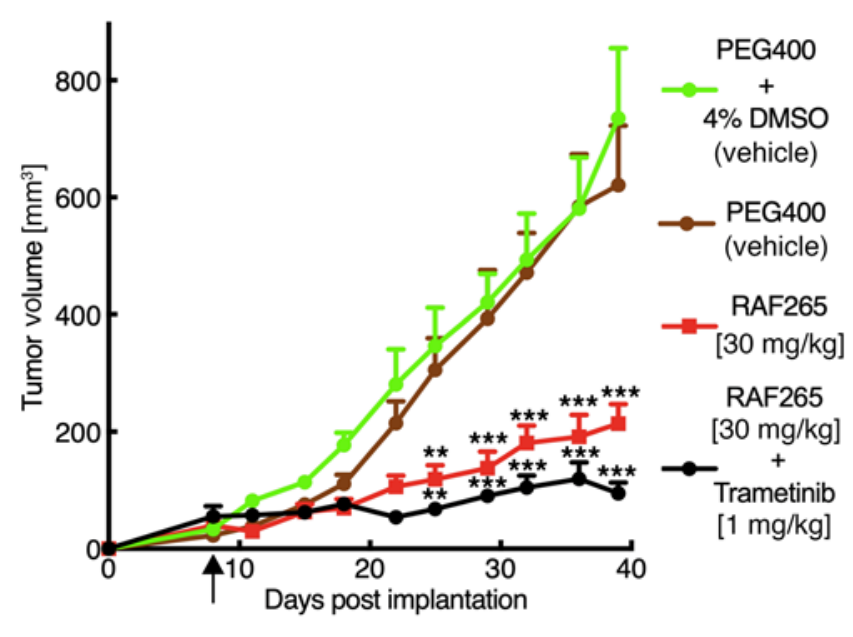

B

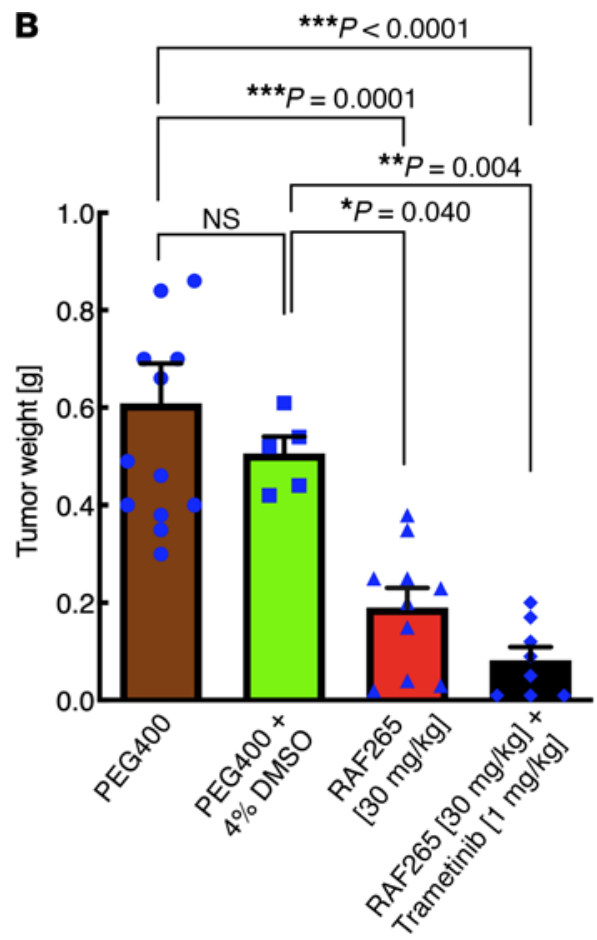

D
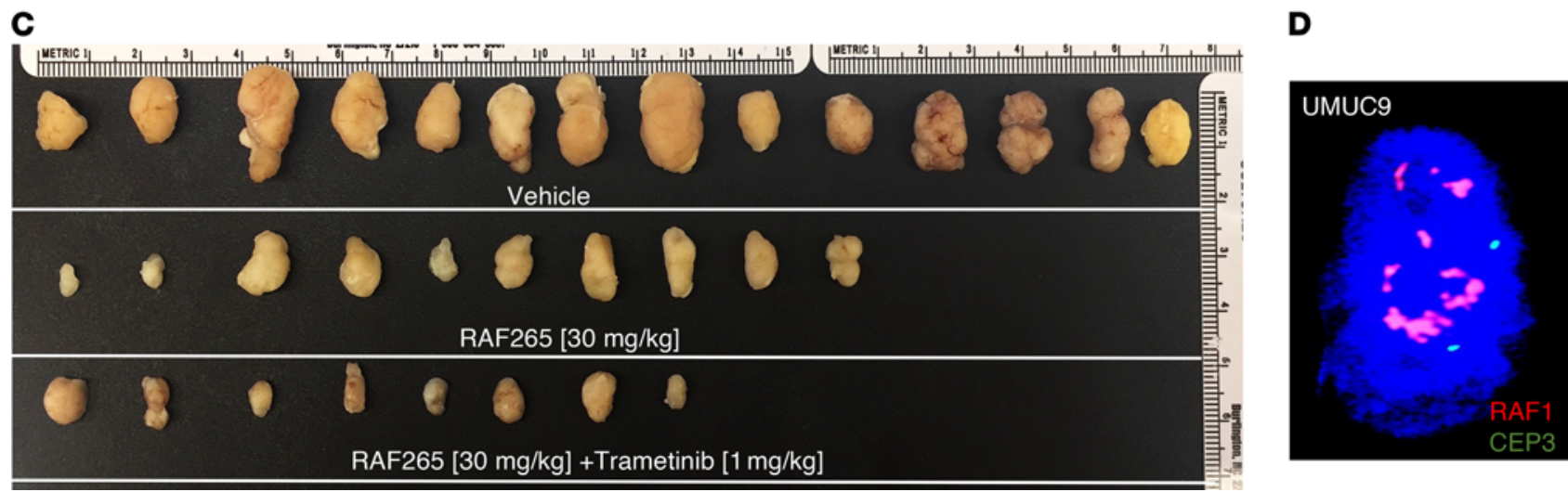

Figure 5. RAF1-amplified tumors are sensitive to RAF and MEK inhibition in vivo. (A) Tumor volumes of UMUC9-engrafted mice treated twice weekly with PEG400 vehicle ( $n=9$ mice), PEG400 with $4 \%$ DMSO vehicle $(n=5)$, RAF265 $(n=10)$, or RAF265 plus trametinib $(n=8)$. The black arrow denotes the day of first treatment. Significant differences in average tumor size are denoted by asterisks. ${ }^{* *} P<0.005$; ${ }^{* *} P<0.0005$, ANOVA with Bonferroni's post hoc test. (B) Mice treated with RAF265 alone or with RAF265 plus trametinib had significantly lower end-of-experiment tumor weights than vehicle-treated mice. Significant differences were calculated by ANOVA with Bonferroni's post hoc test and are denoted by asterisks. ${ }^{*} P<0.05 ;{ }^{* *} P<0.005$; ${ }^{* *} P<0.0005$. (C) Photographs of excised tumors across treatment arms. (D) FISH assay showing RAF1 amplification (red) in UMUC9 tumor xenografts. CEP3 (green) is a chromosome 3 centromeric marker.

luminal subtypes, it is possible that RAF1-amplified tumors may be less likely to respond to chemotherapy than tumors with basal features, as has been observed for FGFR2/3-altered tumors, which are also predominantly luminal (61). Although RAF1amplified and FGFR2/3-altered tumors both have luminal features, we found that RAF1 amplifications and FGFR2/3 alterations are largely mutually exclusive. Therefore, most patients with RAF1-amplified tumors are unlikely to be eligible for an FGFR inhibitor, further highlighting the need for a targeted approach for RAF1-amplified tumors.

A subset of urothelial tumors responds to immune-checkpoint blockade, and anti-PD1/PD-L1 agents are currently approved in the post-cisplatin, cisplatin-ineligible, and switch maintenance settings. The addition of an immune-checkpoint inhibitor to combined BRAF plus MEK inhibition was recently shown to improve outcomes in patients with $\mathrm{BRAF}^{\mathrm{V} 600}$-mutant melanoma, providing evidence for combining MAPK-targeted therapies with immune-checkpoint blockade (62). Numerous clinical trials combining novel RAF inhibitors such as LXH254 with anti-PD 1/PD-L1 agents in patients with advanced solid tumors harboring MAPK pathway alterations are currently ongoing. Additional mechanistic studies will be necessary to define the immunomodulatory effects of RAF inhibitors and other MAPK-targeted agents and to inform optimal combination approaches.

In summary, we characterize a unique subset of urothelial tumors with focal amplification of the $R A F 1$ gene. $R A F 1$-amplified 
A

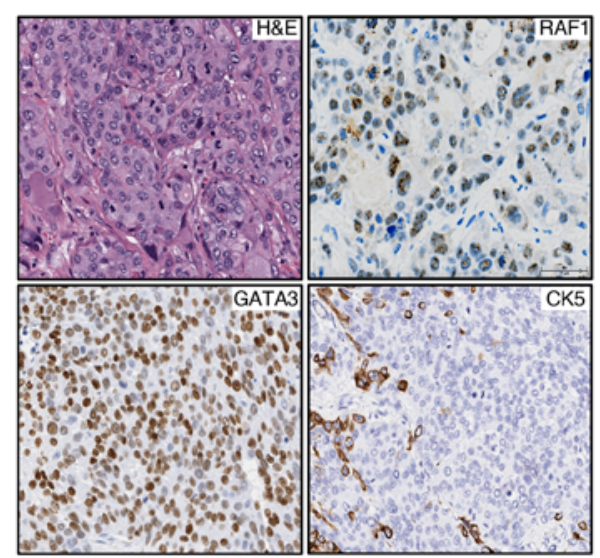

C

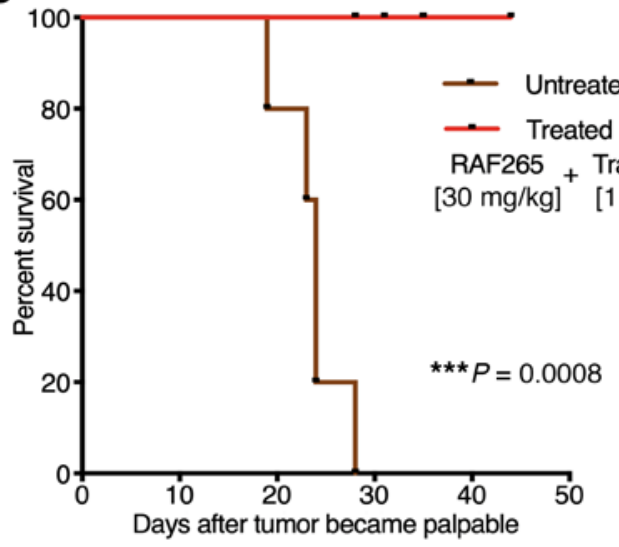

B

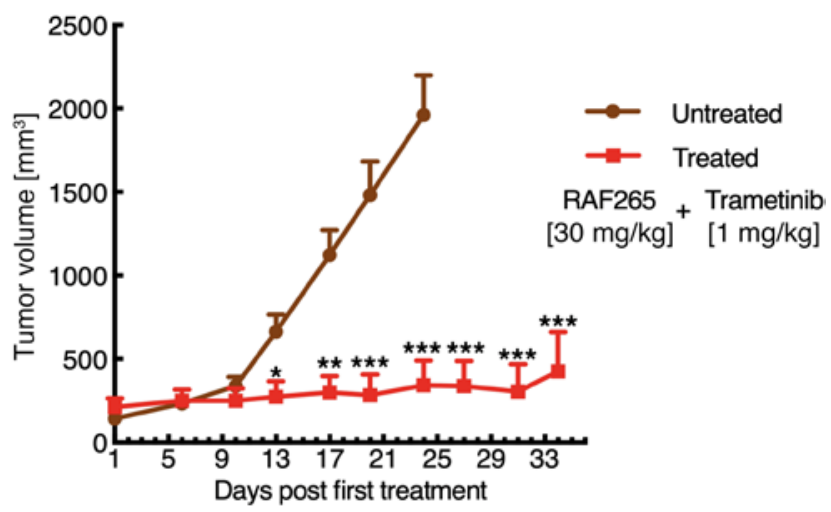

$\mathbf{E}$

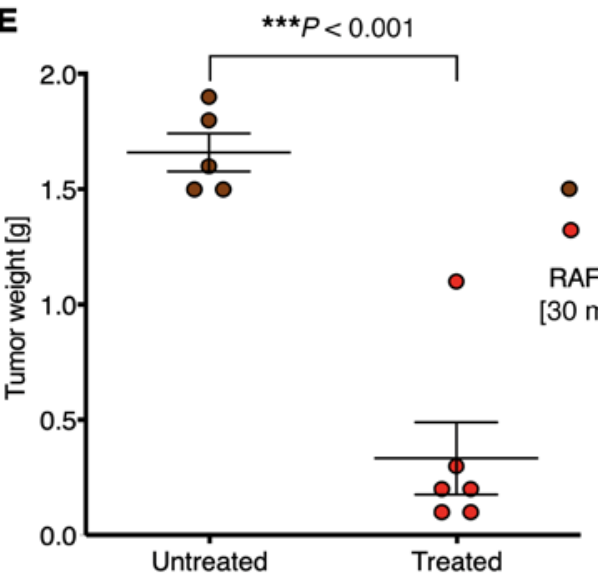

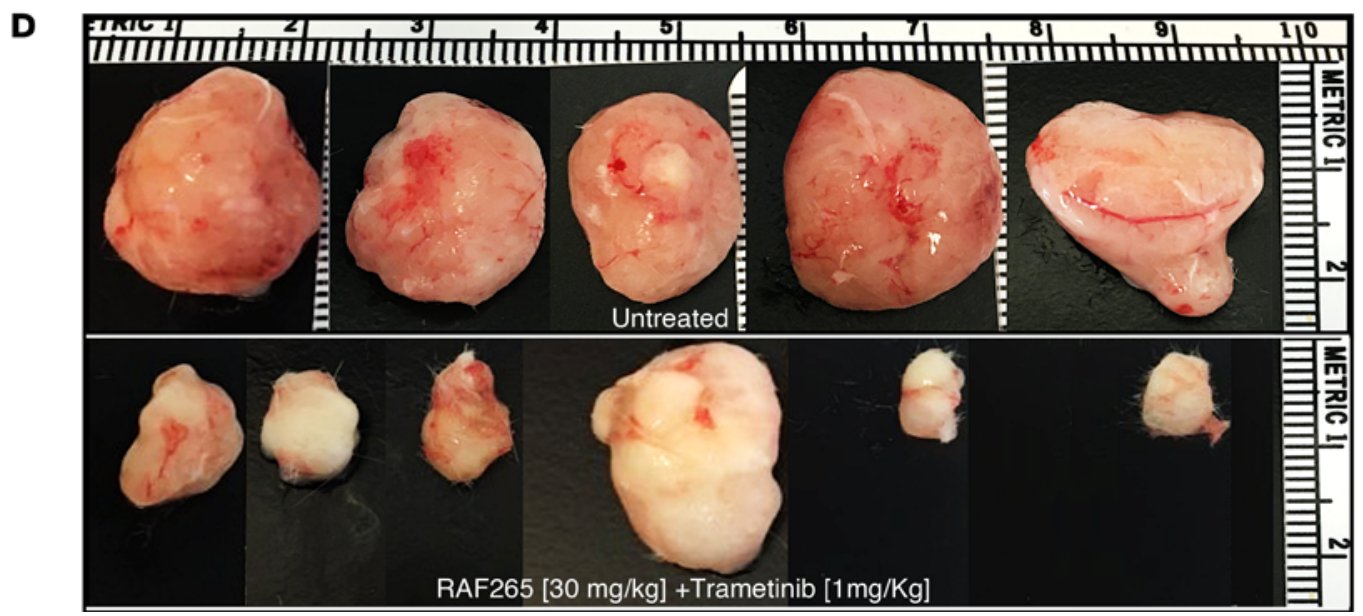

Figure 6. A RAF1-amplified PDX is sensitive to RAF plus MEK inhibition. (A) H\&E and IHC staining for RAF1, the luminal differentiation marker GATA3, and the basal differentiation marker CK5 in a RAF1-amplified PDX tumor show strong RAF1 staining in tumor cells as well as GATA3 staining consistent with a luminal phenotype. Original magnification, $\times 20$ ((H\&E, GATA3 and CK5); $\times 40$ (RAF1). (B) Tumor volume measurements for RAF1-amplified PDX-bearing mice randomized to RAF265 plus trametinib versus no treatment. Significant differences in average tumor size between treated and untreated arms are denoted with asterisks. ${ }^{*} P<0.05 ;{ }^{* *} P<0.005 ;{ }^{* *} P<0.0005$, unpaired 2-tailed Student's $t$ test. (C) Kaplan-Meier survival curves showing percentage of surviving mice in the RAF265 plus trametinib versus untreated arms. Asterisks denote statistical significance by log-rank (Mantel-Cox) test. (D) Photographs of excised tumors from all mice in both arms. (E) The average end-of-experiment tumor weight was significantly lower in the RAF265 plus trametinib-treated mice compared with the untreated mice. Asterisks denote statistical significance by unpaired 2-tailed Student's $t$ test.

urothelial tumors are dependent on RAF1-mediated signaling for survival and display features of luminal differentiation. We show that tumors with activation of MAPK signaling driven by RAF1 amplification or by activating HRAS or NRAS mutations can be targeted with available and emerging RAF inhibitors as well as by combined RAF and MEK inhibition. Together, these data highlight a therapeutic approach for a molecularly defined subset of urothelial tumors. 
A

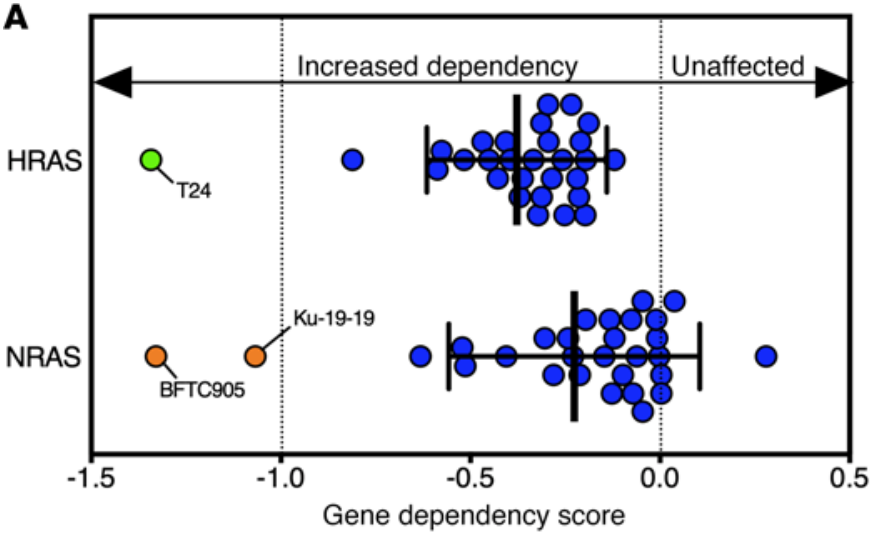

C

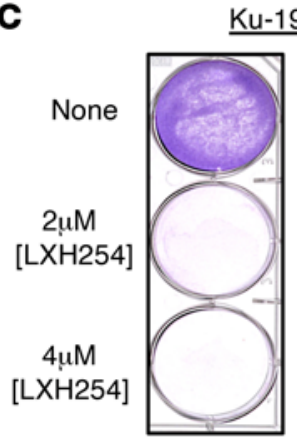

Ku-19-19 (NRAS mut)

D

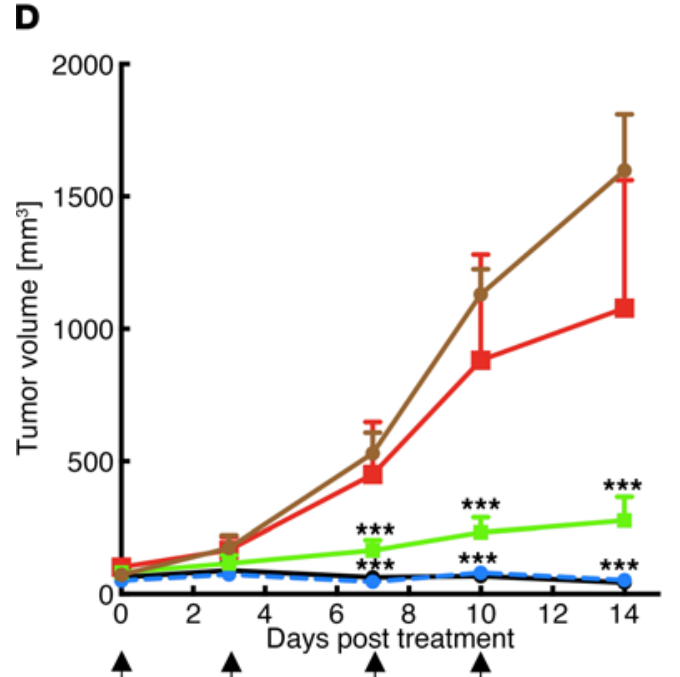

$\left(1^{\text {st }}\right) \quad\left(2^{\text {nd }}\right) \quad\left(3^{\text {rd }}\right) \quad\left(4^{\text {th }}\right)$ drug injections
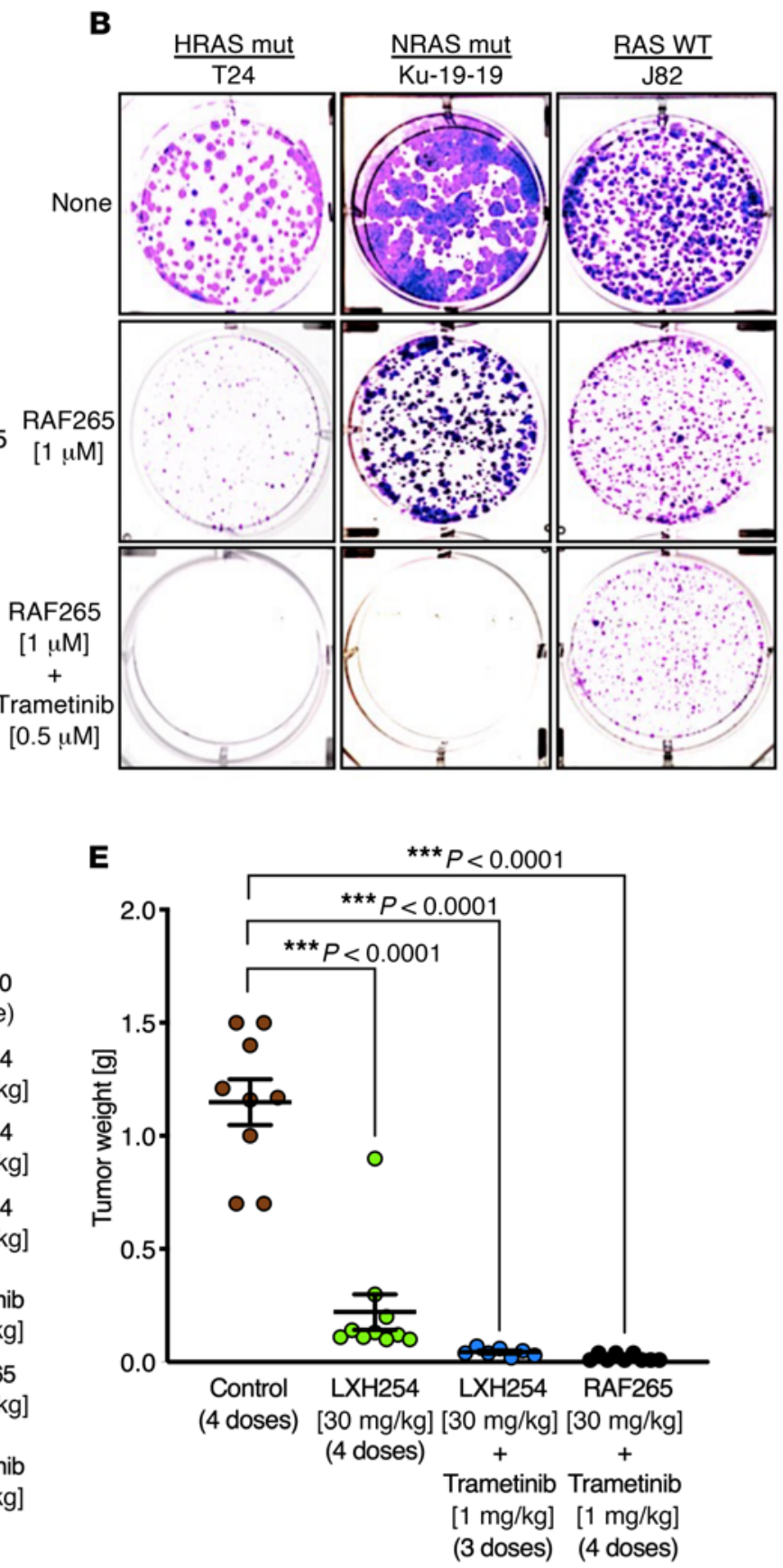

$\mathbf{F}$

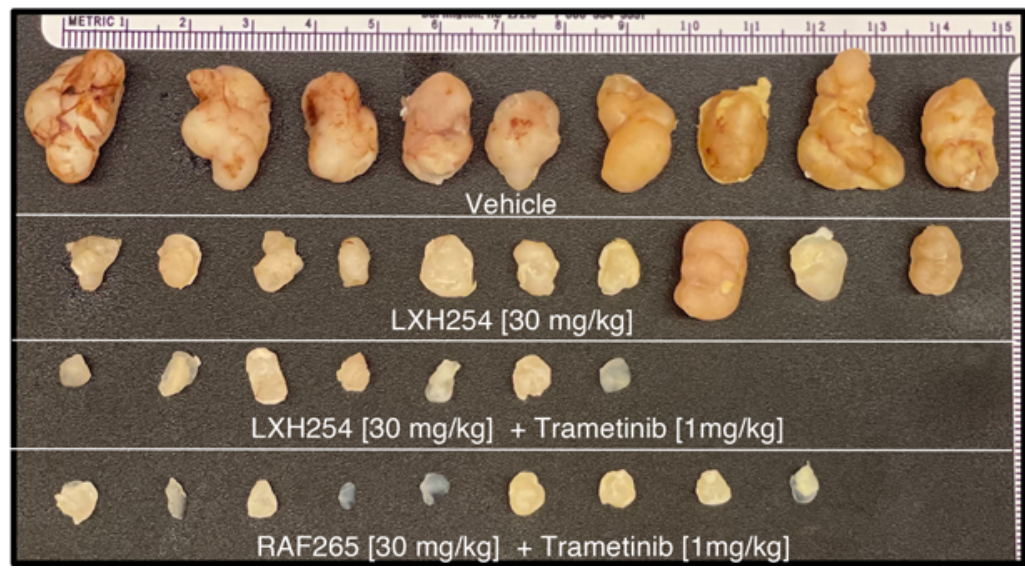


Figure 7. HRAS and NRAS mutant bladder cancer cell lines are sensitive to RAF-targeted therapies. (A) HRAS and NRAS gene-dependency scores from DepMap CRISPR-Cas9 essentiality screens confirm that the HRAS (G12V) mutant T24 bladder cancer cell line shown in green and the NRAS mutant Ku-19-19 (NRAS 061R) and BFTC905 (NRAS Q61L) bladder cancer cell lines shown in orange are dependent on the mutant RAS mutation for survival. (B) Unmagnified colony-formation assays demonstrate increased sensitivity to RAF265 and RAF265 plus trametinib in HRAS-mutant T24 cells and NRAS-mutant Ku-19-19 cells compared with the RAS WT J82 cell line. (C) Crystal violet staining of Ku-19-19 cells 3 days following treatment with LXH254 (left) and immunoblot (blots were run in parallel from the same sample) showing LXH254-induced inhibition of RAF/MEK/ERK signaling (right). (D) Tumor volumes of Ku-19-19-engrafted mice treated twice weekly with PEG400 vehicle ( $n=9$ mice), $15 \mathrm{mg} / \mathrm{kg} \mathrm{LXH256} \mathrm{(} n=5)$, $30 \mathrm{mg} / \mathrm{kg} \mathrm{LXH256} \mathrm{(} n=10), 30 \mathrm{mg} / \mathrm{kg}$ LXH254 plus $1 \mathrm{mg} / \mathrm{kg}$ trametinib $(n=7)$, or $30 \mathrm{mg} / \mathrm{kg}$ RAF265 plus $1 \mathrm{mg} / \mathrm{kg}$ trametinib $(n=9)$. (E) Average end-of-experiment tumor weights for mice treated with vehicle, $30 \mathrm{mg} / \mathrm{kg}$ LXH254, 30 mg/kg LXH254 plus $1 \mathrm{mg} / \mathrm{kg}$ trametinib, or $30 \mathrm{mg} / \mathrm{kg}$ RAF265 plus $1 \mathrm{mg} / \mathrm{kg}$ trametinib. Average tumor weights were significantly lower in all treatment arms compared with those of vehicle-treated tumors. Significant differences in average tumor size and weight in the treatment groups compared with vehicle are denoted with asterisks. ${ }^{* *} P<0.0001$, ANOVA with Bonferroni's post hoc test. (F) Photographs of excised tumors.

\section{Methods}

\section{Genomic analyses}

TCGA. Data for DNA (copy number alterations, mutations, and fusions), gene expression (RNA Seq V2 RSEM), and RPPA protein expression were extracted for the TCGA BLCA cohort from the Broad Institute Firehose Legacy and PanCancer Atlas data sets (28). Genomic data visualization and gene expression heatmaps were generated with OncoPrinter from cBioportal $(63,64)$. Focal chromosomal copy number plots were from SNP6 copy number analysis (GISTIC2) output (65) and acquired from the Broad Institute's TCGA Genome Data Analysis Center (FireBrowse).

DepMap. The DepMap includes publicly available data sets from the Cancer Cell Line Encyclopedia (CCLE) $(31,32)$, genome-wide dependency screens (33), and the Cancer Therapeutics Response Portal (CTRP) (66). DNA copy number, gene expression, mutation, and gene-dependency scores for all available bladder cancer cell lines as well as relevant drug sensitivity data were downloaded from the DepMap portal.

OncoPanel. OncoPanel is a cancer genomic assay performed using DNA extracted from fresh-frozen or FFPE tumor samples from cancer patients treated at Dana-Farber Cancer Institute. OncoPanel is designed to detect somatic mutations, copy number variations, and structural variants in a curated set of 447 cancer-related genes (67-69).

\section{IHC}

Paraffin processing, embedding, and sectioning were performed at the Specialized Histopathology Core at the Dana-Farber Cancer Institute/Harvard Cancer Center. FFPE tumor tissues were sectioned from paraffin blocks and mounted on glass slides. IHC was performed on the Leica Bond III automated staining platform using the Leica Biosystems Refine Detection Kit with citrate antigen retrieval. Primary antibodies were used for RAF1 (1:50; polyclonal, catalog HPA002640, MilliporeSigma), anti-phospho-histone H2A.X (1:50; clone JBW301, MilliporeSigma), cleaved PARP (1:50; clone D64E10, CST), Ki67 (1:50; clone SP6, Biocare Medical), CK5 (1:500, clone EP1601Y, Abcam), and GATA3 (1:100; clone D13C9, CST).

\section{FISH assay}

The FISH assay was performed at the Brigham and Women's Hospital's Cytogenomics Core Laboratory. Thin sections from FFPE tumor tissue were mounted on glass slides. The slides were warmed to $60^{\circ} \mathrm{C}$ and deparaffinized in xylene at room temperature for 15 minutes, followed by immersion in $100 \%$ ethanol for 2 minutes. The slides were immersed in $100^{\circ} \mathrm{C} 100 \mathrm{mM}$ Tris-base with $50 \mathrm{mM}$ EDTA (pH 7.0) and then continually heated at $100^{\circ} \mathrm{C}$ in a microwave for 45 minutes. The slides were subsequently washed in PBS for 5 to 10 minutes, and $150 \mu \mathrm{L}$ of DigestAll (Zymed) was then added to each slide before covering with a plastic coverslip. Slides were placed in a dry oven at $37^{\circ} \mathrm{C}$ for 20 minutes, and Digest-All was then added for a second time and the step was repeated before washing with PBS for 5 to 10 minutes. The slides were sequentially dehydrated in $70 \%, 90 \%$, and $100 \%$ ethanol at room temperature for 2 minutes each and air-dried. A $22 \times 22 \mathrm{~mm}$ area was marked on each slide and 8 to $10 \mu \mathrm{L}$ of RAF1 and CEP3 (chromosome 3 centromere) probes was added to each slide. Probes were purchased from Empire Genomics. After staining, slides were covered with a plastic coverslip and heated at $94^{\circ} \mathrm{C}$ for 3 minutes to codenature and allow hybridization of the probe to genomic DNA. The slides were incubated in a humidified chamber at $37^{\circ} \mathrm{C}$ for 2 days. The slides were then immersed in $0.5 \times$ SSC buffer $(75$ $\mathrm{mM} \mathrm{NaCl}$ and $7.5 \mathrm{mM}$ sodium citrate; $\mathrm{pH} 7.0$ ) and heated to $72^{\circ} \mathrm{C}$ for 5 minutes, followed by washing 3 times in PBS-Tween (0.025\%) for 2 minutes each at room temperature. After this step, 8 to $10 \mu \mathrm{L}$ of DAPI was added, a glass coverslip was applied to each slide, and the slides were viewed under a fluorescence microscope for image acquisition.

\section{Cell lines and reagents}

Bladder cancer cell lines were purchased from ATCC. Cells were maintained in RPMI 1640 medium supplemented with fetal bovine serum, penicillin/streptomycin, and L-glutamine (Life Technologies) unless otherwise specified. Inhibitors (RAF265/CHIR-265, Trametinib/ GSK112021, and LXH254) were purchased from Selleck Chemicals. Formalin (neutral buffered, 10\%), crystal violet solution, protease inhibitor cocktail, and phosphatase inhibitor (PhosSTOP) were purchased from MilliporeSigma. Polyethylene Glycol 400 (PEG400) was purchased from Fisher Scientific. Matrigel was purchased from BD Biosciences. Dicer-substrate siRNAs targeting RAF1 as well as a nontargeting control siRNA (siNTC) were purchased from Integrated DNA Technologies Inc. The sequences for RAF1 siRNA no. 1 and no. 2 are provided in Supplemental Table 7.

\section{siRNA gene depletion}

Cells were seeded in 6-well plates and grown to 50\% confluence. A transfection mix was prepared by combining Lipofectamine 3000 (Life Technologies) with either siNTC or siRAF1 in Opti-MEM reduced serum medium (Life Technologies) according to the manufacturer's protocol. The transfection mix was then added to the growth medium to a final siRNA concentration of $30 \mathrm{nM}$, which was then added to each well. After 48 hours, cells were trypsinized and equal numbers of cells were aliquoted to separate wells for a second round of siRNA transfection with $15 \mathrm{nM}$ siNTC or siRAF1. After 24 hours, the media were replaced and cells were allowed to grow for 3 days prior to cell viability analysis.

\section{Cell viability and proliferation assays}

The CellTiter-Glo luminescence assay was performed by seeding cells in either a 96-well (2500-5000 cells/well) or 24-well (20,000 cells/ 
well) format. The drug was diluted in media and added to the wells the following day. Three days later, media were removed and CellTiter-Glo reagent (Promega) was added according to the manufacturer's instruction. The intensity of luminescent signal was measured using a luminescence microplate reader (BioTek). Relative viability at each drug concentration was calculated by dividing the signal intensity from treated wells by that from mock-treated wells from 3 independent experiments. Synergy analysis for drug combinations was calculated from the cell-viability data using Combenefit software (70). For crystal violet staining experiments, cells were seeded in 6-well plates at 50,000 to 100,000 cells/well for 3-day experiments or 1000 cells/well for 10- to 14-day colony-formation assays. Vehicle or drug (diluted in media) was added the day after plating. At the end of the experiment, media was removed and cells were fixed in formalin solution for 30 minutes and then stained with crystal violet prepared in equal volumes of methanol and water. Excess crystal violet was removed by washing with PBS, and plates were then dried and imaged using an Amersham imager; and signal intensity was quantified with ImageJ software (NIH). To assess the effect of treatments on cell proliferation, cell number was counted before and after treatment using a Countess Automated Cell Counter from Life Technologies according to the manufacturer's instructions. To assess apoptosis, the level of activated caspase- $3 / 7$ was measured before and after drug treatment using the Caspase-Glo 3/7 Assay Kit (Promega) according to the manufacturer's instructions.

\section{Immunoblots}

RIPA buffer supplemented with protease and phosphatase inhibitors (Roche) was used to make cell lysates. Samples were then sonicated, and the protein amount in each sample was determined using the Bradford assay. Sample buffer (Bio-Rad) was then added to equal amounts of protein, followed by denaturation of the lysates at $90^{\circ} \mathrm{C}$ for 10 minutes. Samples were then loaded on NuPAGE protein gels (Thermo Fisher Scientific). The gel was subsequently transferred to nitrocellulose membrane in a NuPAGE transfer buffer. At the end of the transfer, the membrane was blocked in 5\% milk in TBS buffer for 30 minutes. Sections of the membrane corresponding to the desired protein molecular weights were stained overnight with primary antibodies: RAF1 (1:1000; catalog 9422, CST), BRAF (1:1000; clone D9T6S, CST), ERK1/2 (1:1000; clone 3A7, CST), AKT (1:1000; clone C67E7, CST), phospho-MEK1/2 (1:1000; clone 41G9, CST), phospho-ERK1/2 (1:1000; clone D13.14.4E, CST), phospho-AKT(1:1000; clone D9E, CST), total and cleaved PARP (1:1000, catalog 9542, CST), phospho-H2AX Ser139 (1:1000, clone JBW301, MilliporeSigma), or $\beta$-tubulin (1:1000, clone G-8, Santa Cruz Biotechnology) prepared in 1\% milk in TBST. A LI-COR Odyssey Infrared Imaging System was used for signal detection using IRDYE-conjugated secondary antibodies (LI-COR Biosciences). See complete unedited blots in the supplemental material.

\section{Microscopy}

All phase contrast microscopy images were obtained at either $\times 4$ or $\times 10$ magnification using a Zeiss Primo-Vert Inverted Phase Contrast Microscope. Images were acquired from 3 nonoverlapping fields for each sample from 3 independent experiments.

\section{Cell line xenograft experiments}

Six-week-old female athymic nude mice, NU/J (stock no. 002019) homozygous for Foxn1 $1^{\text {nu }}$, were purchased from Jackson Laboratory.
Mice were housed at Dana-Farber's Animal Resources Facility. At 7 to 9 weeks of age, mice were anesthetized with isoflurane and subcutaneously injected on the left flank with 3 million UMUC9 cells or 1 million Ku-19-19 cells prepared 1:1 with Matrigel (BD Biosciences) and PBS in a total volume of $200 \mu \mathrm{L}$. Tumor growth was measured twice weekly with a digital caliper and calculated using the formula $L$ $\left.\times W^{2}\right) \times 1 / 2, L$ indicates length and $W$ indicates width. When tumors reached an average volume of approximately $100 \mathrm{~mm}^{3}$, mice were randomized to treatment or control arms. RAF265 and LXH254 were prepared in PEG400 to a stock concentration of $6 \mathrm{mg} / \mathrm{mL}$ and were delivered i.p. twice weekly at doses of $30 \mathrm{mg} / \mathrm{kg}$ for a total of 9 doses. For mice receiving combination treatment, trametinib was prepared in PEG400 containing 4\% DMSO at a concentration of $0.2 \mathrm{mg} / \mathrm{mL}$ in addition to RAF265/LXH254 at $6 \mathrm{mg} / \mathrm{mL}$ and delivered to mice at a dose of $30 \mathrm{mg} / \mathrm{kg}$ RAF265/LXH254 plus $1 \mathrm{mg} / \mathrm{kg}$ trametinib. Mice in the control group were injected with either PEG400 alone or PEG400 containing $4 \%$ DMSO. The health status of each mouse was monitored daily, and weights were recorded twice weekly. For UMUC9 xenografts, mice were sacrificed following completion of 9 doses. For Ku-19-19 xenografts, mice received a total of 4 doses and the study was ended 14 days after the first drug dose, as all mice in the control arm had reached the protocol-defined end point.

\section{PDX experiments}

The Mouse Models of Human Cancer database (MMHCdb) (71) includes genomic data from hundreds of human PDX tumors that are banked and distributed by The Jackson Laboratory. We used this database to identify a PDX model derived from a patient with high-grade urothelial carcinoma of the bladder (catalog TM00024/BL0440F). The TM00024 model had the highest RAF1 gene expression level across available bladder tumor PDXs, and its RAF1 amplification has been previously confirmed (39). Two NSG mice bearing the TM00024 model were purchased from The Jackson Laboratory. Both mice were sacrificed, and the freshly harvested tumor tissue was combined (totaling approximately $700 \mathrm{~mm}^{3}$ ) and was minced aseptically using a sterile scalpel into a Petri dish containing fresh RPMI-1640 media. Prior to mincing, any surrounding nontumor tissue was carefully removed and the remaining tumor tissue was closely inspected to ensure any grossly necrotic areas were discarded. The minced tumor tissue was then evenly aliquoted into prechilled tubes. Six-week-old NSG mice, strain NOD. Cg-Prkdc ${ }^{\text {scid }}$ Il2 rg $^{\text {tm1Wil }} /$ SzJ (stock no. 005557), were purchased from The Jackson Laboratory. At 7 weeks of age, mice were anesthetized with isoflurane and the freshly minced tumor tissue was mixed with Matrigel $(100 \mu \mathrm{L})$ and injected subcutaneously into a small incision made on the left flank. The majority of implanted mice developed palpable tumors by day 45 , and mice were randomized to treatment or control group when tumors measured at least $100 \mathrm{~mm}^{3}$. Mice in the treatment group received an i.p. dose of $30 \mathrm{mg} / \mathrm{kg}$ RAF265 plus $1 \mathrm{mg} / \mathrm{kg}$ trametinib twice weekly for a maximum of 9 doses. Tumor-bearing mice were sacrificed following completion of 9 doses or when tumor size reached a protocol-defined end point of $2 \mathrm{~cm}$ in the greatest dimension.

\section{Statistics}

All results are reported as mean \pm SEM of 3 or more independent experiments. $P$ values were calculated using Student's $t$ test or the ANOVA test for multiple comparisons. For Kaplan-Meier survival analysis, the logrank (Mantel-Cox) test was used to determine significance. GraphPad 
Prism was used to generate graphs and calculate statistics. Significance values of $P<0.05$ were considered statistically significant.

\section{Study approval}

Institutional patient samples. Urothelial cancer cases were identified from patients at Dana-Farber Cancer Institute and Brigham and Women's Hospital. Tumor specimen and clinicopathologic information were collected with IRB approval (DFCI IRB protocol 11-104), and clinical and genomic characteristics of the entire cohort $(n=472)$ have been recently described (29). Clinicopathologic characteristics based on RAF1 and HRAS status are summarized in Supplemental Tables 2 and 4.

Animal study. Mice were housed at Dana-Farber's Animal Resources Facility, and all animal experiments were performed in accordance with an IACUC-approved animal protocol (no. 18-002).

\section{Author contributions}

RTB and KWM conceived and designed the project. RTB and ASS performed in vitro and in vivo experiments. RTB, JS, DLM, and JHH performed genomic analysis of TCGA samples. RTB, AHN, and CRC analyzed and interpreted clinical data from the Dana-Farber Cancer Institute/Brigham patient cohort. EPG, AN, and ART performed OncoPanel analysis from institutional samples. J Börcsök and ZS performed bioinformatic analyses to infer clonality of RAF1-amplified samples. RTB and KWM wrote the manuscript. KWM supervised this study. J Bellmunt, CXP, DJK, GPS, EMVA, RTB, ASS, JS, DLM, JHH, AHN, CRC, EPG, AN, ART, J Börcsök, ZS, and KWM discussed the findings and edited the manuscript.

\section{Acknowledgments}

We thank members of the Mouw lab for insights and discussion on all aspects of the work. We thank Dana-Farber Cancer Institute/ Harvard Cancer Center for the use of the Specialized Histopathology Core, which provided histology and immunohistochemistry services. Dana-Farber Cancer Institute/Harvard Cancer Center is supported in part by National Cancer Institute Cancer Center support grant NIH 5 P30 CA06516. Support was received from National Cancer Institute/NIH K08 CA219504 and Burroughs Wellcome Career Award for Medical Scientists (to KWM). We thank the Brigham and Women's Hospital's Cytogenomics Core Laboratory for assistance with RAF1 FISH experiments.

Address correspondence to: Kent W. Mouw, Dana-Farber Cancer Institute, 450 Brookline Ave., HIM328, Boston, Massachusetts 02215, USA. Phone: 617.582.9356; Email: kent_mouw@dfci.harvard.edu.
1. Siegel RL, et al. Cancer statistics, 2020. CA Cancer JClin. 2020;70(1):7-30.

2. Robertson AG, et al. Comprehensive molecular characterization of muscle-invasive bladder cancer. Cell. 2017;171(3):540-556.

3. Kamoun A, et al. A consensus molecular classification of muscle-invasive bladder cancer. Eur Urol. 2020;77(4):420-433.

4. Powles T, et al. Avelumab maintenance therapy for advanced or metastatic urothelial carcinoma. N Engl JMed. 2020;383(13):1218-1230.

5. Bellmunt J, et al. Pembrolizumab as second-line therapy for advanced urothelial carcinoma. N Engl JMed. 2017;376(11):1015-1026.

6. Glaser AP, et al. The evolving genomic landscape of urothelial carcinoma. Nat Rev Urol. 2017;14(4):215-229.

7. Loriot Y, et al. Erdafitinib in locally advanced or metastatic urothelial carcinoma. $N$ Engl J Med. 2019;381(4):338-348.

8. Sturgill TW, et al. Insulin-stimulated MAP-2 kinase phosphorylates and activates ribosomal protein S6 kinase II. Nature. 1988;334(6184):715-718.

9. Dhanasekaran N, Reddy EP. Signaling by dual specificity kinases. Oncogene. 1998;17(11):1447-1455.

10. Durrant DE, Morrison DK. Targeting the Raf kinases in human cancer: the Raf dimer dilemma. Br JCancer. 2018;118(1):3-8.

11. Drosten M, Barbacid M. Targeting the MAPK pathway in KRAS-driven tumors. Cancer Cell. 2020;37(4):543-550.

12. Desideri E, et al. Alike but different: RAF paralogs and their signaling outputs. Cell. 2015;161(5):967-970.

13. Matallanas D, et al. Raf family kinases: old dogs have learned new tricks. Genes Cancer. 2011;2(3):232-260.

14. Karoulia Z, et al. New perspectives for targeting RAF kinase in human cancer. Nat Rev Cancer. 2017;17(11):676-691.
15. Akbani R, et al. Genomic classification of cutaneous melanoma. Cell. 2015;161(7):1681-1696.

16. Kimura ET, et al. High prevalence of BRAF mutations in thyroid cancer: genetic evidence for constitutive activation of the RET/PTC-RAS-BRAF signaling pathway in papillary thyroid carcinoma. Cancer Res. 2003;63(7):1457.

17. Weber CK, et al. Active Ras induces heterodimerization of $\mathrm{cRaf}$ and BRaf. Cancer Res. 2001;61(9):3595-3598.

18. Freeman AK, et al. The importance of Raf dimerization in cell signaling. Small GTPases. 2013;4(3):180-185.

19. Hu J, et al. Allosteric activation of functionally asymmetric RAF kinase dimers. Cell. 2013;154(5):1036-1046.

20. Rajakulendran T, et al. A dimerization-dependent mechanism drives RAF catalytic activation. Nature. 2009;461(7263):542-545.

21. Flaherty KT, et al. Inhibition of mutated, activated BRAF in metastatic melanoma. NEngl J Med. 2010;363(9):809-819.

22. Chapman $P B$, et al. Improved survival with vemurafenib in melanoma with BRAF V600E mutation. N Engl J Med. 2011;364(26):2507-2516.

23. Flaherty KT, et al. Combined BRAF and MEK inhibition in melanoma with BRAF V600 mutations. N Engl J Med. 2012;367(18):1694-1703.

24. Roviello G, et al. Encorafenib, binimetinib, and cetuximab in BRAF V600E-mutated colorectal cancer. Transl Oncol. 2020;13(9):876.

25. Xue JY, Lito P. Quest for clinically effective RAF dimer inhibitors. JClin Oncol. 2020;38(19):2197-2200.

26. Yaeger R, Corcoran RB. Targeting alterations in the RAF-MEK pathway. Cancer Discov. 2019;9(3):329-341.

27. Peng SB, et al. Inhibition of RAF isoforms and active dimers by LY3009120 leads to anti-tumor activities in RAS or BRAF mutant cancers. Cancer
Cell. 2015;28(3):384-398.

28. Hoadley KA, et al. Cell-of-origin patterns dominate the molecular classification of 10,000 tumors from 33 types of cancer. Cell. 2018;173(2):291-304

29. Nassar AH et al. Mutational analysis of 472 urothelial carcinoma across grades and anatomic sites. Clin Cancer Res. 2019;25(8):2458-2470.

30. Sjödahl G, et al. Molecular profiling in muscle-invasive bladder cancer: more than the sum of its parts. J Pathol. 2019;247(5):563-573.

31. Ghandi M, et al. Next-generation characterization of the Cancer Cell Line Encyclopedia. Nature. 2019;569(7757):503-508.

32. Barretina J, et al. The Cancer Cell Line Encyclopedia enables predictive modelling of anticancer drug sensitivity. Nature. 2012;483(7391):603-607.

33. Meyers RM, et al. Computational correction of copy number effect improves specificity of CRISPRCas9 essentiality screens in cancer cells. Nat Genet. 2017;49(12):1779-1784.

34. Williams TE, et al. Discovery of RAF265: a potent mut-B-RAF inhibitor for the treatment of metastatic melanoma. ACS Med Chem Lett. 2015;6(9):961-965.

35. Tsai J, et al. Discovery of a selective inhibitor of oncogenic B-Raf kinase with potent antimelanoma activity. Proc Natl Acad Sci US A. 2008;105(8):3041-3046.

36. Long GV, et al. Combined BRAF and MEK inhibition versus BRAF inhibition alone in melanoma. N Engl J Med. 2014;371(20):1877-1888.

37. Su Y, et al. RAF265 inhibits the growth of advanced human melanoma tumors. Clin Cancer Res. 2012;18(8):2184-2198.

38. Izar B, et al. A first-in-human phase I, multicenter, open-label, dose-escalation study of the oral RAF/VEGFR-2 inhibitor (RAF265) in locally advanced or metastatic melanoma independent from BRAF mutation status. Cancer Med. 2017;6(8):1904-1914. 
39. Pan CX, et al. Development and characterization of bladder cancer patient-derived xenografts for molecularly guided targeted therapy. PLoS One. 2015;10(8):1-22.

40. Uhlen M, et al. A pathology atlas of the human cancer transcriptome. Science. 2017;357(6352):eaan2507.

41. Terrell EM, et al. Distinct binding preferences between Ras and Raf family members and the impact on oncogenic Ras signaling. Mol Cell. 2019;76(6):872-884.

42. Necchi A, et al. Comprehensive genomic profiling of upper-tract and bladder urothelial carcinoma [published online August 26, 2020]. Eur Urol Focus. https://doi.org/10.1016/j.euf.2020.08.001.

43. Kim K, et al. Modeling biological and genetic diversity in upper tract urothelial carcinoma with patient derived xenografts. Nat Commun. 2020;11(1):1975.

44. Fujii Y, et al. Molecular classification and diagnostics of upper urinary tract urothelial carcinoma. Cancer Cell. 2021;39(6):793-809.

45. Ramurthy $S$, et al. Design and discovery of $N$-(3-(2-(2-Hydroxyethoxy)-6-morpholinopyridin-4-yl)-4-methylphenyl)-2-(trifluoromethyl) isonicotinamide, a selective, efficacious, and well-tolerated RAF inhibitor targeting RAS mutant cancers: the path to the clinic. JMed Chem. 2020;63(5):2013-2027.

46. Janku F, et al. A phase I study of LXH254 in patients (pts) with advanced solid tumors harboring MAPK pathway alterations. J Clin Oncol. 2018;36(15_suppl):2586.

47. Simon R, et al. High-throughput tissue microarray analysis of 3p25 (RAF1) and 8p12 (FGFR1) copy number alterations in urinary bladder cancer. Cancer Res. 2001;61(11):4514-4519.

48. Varley CL, et al. Role of PPARgamma and EGFR signalling in the urothelial terminal differentiation programme. J Cell Sci. 2004;117(10):2029-2036.

49. Goldstein JT, et al. Genomic activation of PPARG reveals a candidate therapeutic axis in bladder cancer. Cancer Res. 2017;77(24):6987-6998.
50. Misra P, et al. Phosphorylation of transcriptional coactivator peroxisome proliferator-activated receptor (PPAR)-binding protein (PBP). Stimulation of transcriptional regulation by mitogen-activated protein kinase. J Biol Chem. 2002;277(50):48745-48754.

51. Decker B, et al. Homologous mutation to human BRAF V600E is common in naturally occurring canine bladder cancer--evidence for a relevant model system and urine-based diagnostic test. Mol Cancer Res. 2015;13(6):993-1002.

52. Yao Z, et al. RAF inhibitor PLX8394 selectively disrupts BRAF dimers and RAS-independent BRAF-mutant-driven signaling. Nat Med. 2019;25(2):284-291.

53. Wang J, et al. A secondary mutation in $B R A F$ confers resistance to RAF inhibition in a $B R A F^{\mathrm{V} 600 \mathrm{E}}$-mutant brain tumor. Cancer Discov. 2018;8(9):1130-1141.

54. Desai J, et al. Phase I, open-label, dose-escalation/ dose-expansion study of lifirafenib (BGB-283), an RAF family kinase inhibitor, in patients with solid tumors. J Clin Oncol. 2020;38(19):2140-2150.

55. Olszanski AJ, et al. Phase I study of the investigational, oral pan-RAF kinase inhibitor TAK-580 (MLN2480) in patients with advanced solid tumors (ST) or melanoma (MEL): final analysis. Ann Oncol. 2017;28(suppl 5):136-137.

56. Kim TW, et al. Belvarafenib, a novel pan-RAF inhibitor, in solid tumor patients harboring BRAF, KRAS, or NRAS mutations: phase I study. JClin Oncol. 2019;37(15_suppl):3000.

57. Hu Y, et al. Abstract 4038: ABM-1310, a novel BRAF inhibitor, combined with EGFR and MEK inhibitors, inhibits colorectal tumor growth and increases overall survival in vivo. Paper presented at: AACR Annual Meeting 2020; April 27-28 and June 22-24, 2020; Philadelphia, Pennsylvania, USA. Accessed September 21, 2021.

58. Karaman MW et al. A quantitative analysis of kinase inhibitor selectivity. Nat Biotechnol. 2008;26(1):127-132.

59. Su F et al. RAS mutations in cutaneous squa- mous-cell carcinomas in patients treated with BRAF inhibitors. N Engl JMed. 2012;366(3):207-215.

60. Seiler R et al. Impact of molecular subtypes in muscle-invasive bladder cancer on predicting response and survival after neoadjuvant chemotherapy. Eur Urol. 2017;72(4):544-554.

61. Teo MY, et al. Fibroblast growth factor receptor 3 alteration status is associated with differential sensitivity to platinum-based chemotherapy in locally advanced and metastatic urothelial carcinoma. Eur Urol. 2020;78(6):907-915.

62. Ascierto PA, et al. Dabrafenib, trametinib and pembrolizumab or placebo in BRAF-mutant melanoma. Nat Med. 2019;25(6):941-946.

63. Gao J, et al. Integrative analysis of complex cancer genomics and clinical profiles using the cBioPortal. Sci Signal. 2013;6(269):pl1.

64. Cerami E, et al. The cBio cancer genomics portal: an open platform for exploring multidimensional cancer genomics data. Cancer Discov. 2012;2(5):401-404.

65. Mermel CH, et al. GISTIC2.0 facilitates sensitive and confident localization of the targets of focal somatic copy-number alteration in human cancers. Genome Biol. 2011;12(4):R41.

66. Basu A, et al. An interactive resource to identify cancer genetic and lineage dependencies targeted by small molecules. Cell. 2013;154(5):1151-1161.

67. Sholl LM, et al. Institutional implementation of clinical tumor profiling on an unselected cancer population. JCI Insight. 2016;1(19):e87062.

68. Garcia EP, et al. Validation of OncoPanel: a targeted next-generation sequencing assay for the detection of somatic variants in cancer. Arch Pathol Lab Med. 2017;141(6):751-758.

69. Hanna GJ et al. Frameshift events predict antiPD-1/L1 response in head and neck cancer. JCI Insight. 2018;3(4):e98811.

70. Di Veroli GY, et al. Combenefit: an interactive platform for the analysis and visualization of drug combinations. Bioinformatics. 2016;32(18):2866-2868.

71. Krupke DM, et al. The mouse tumor biology database. Nat Rev Cancer. 2008;8(6):459-465 